Acta Univ. Sapientiae, Mathematica, 12, 2 (2020) 317-346

DOI: $10.2478 /$ ausm-2020-0023

\title{
Soft covered ideals in semigroups
}

\author{
Şerif Özlü \\ Department of Mathematics \\ Gaziantep University \\ 27300 Gaziantep, Turkey \\ email: serif.ozlu@hotmail.com
}

\author{
Aslihan Sezgin \\ Department of Mathematics \\ and Science Education \\ Amasya University \\ 05100 Amasya, Turkey \\ email: aslihan.sezgin@amasya.edu.tr
}

\begin{abstract}
In this work, soft covered ideals in semigroups are constructed and in this concept, soft covered semigroups, soft covered left (right) ideals, soft covered interior ideals, soft covered (generalized) bi-ideals and soft covered quasi ideals of a semigroup are defined. Various properties of these ideals are introduced and the interrelations of these soft covered ideals and the relations of soft anti covered ideals and soft covered ideals are investigated.
\end{abstract}

\section{Introduction}

Soft set theory introduced by Molodstov in 1999 [1] is applied uncertainness complicated problems especially in economy, medical, engineering and over classical Mathematics such as groups [2], semirings [3], rings [4], BCK/BCIalgebras [5, 6, 7], d-algebras [8], BL-algebras [9], BCH-algebras [10] and nearrings [11].

The works over soft set theory progressed rapidly and after then, this theory started to extend over fuzzy set, rough set and so on and a lot of mathematical structures were constructed over these sets. Moreover, soft set theory has

2010 Mathematics Subject Classification: Primary 03EE99; Secondary 03E75 Key words and phrases: soft set, soft covered left(right) ideal, soft covered ideal, soft covered bi-ideal, soft covered interior ideal, soft covered quasi-ideal 
received abroad attention by several authors in game theory, intelligent system and especially decision making method. After decision making method was applied over uncertainness sets, it could solve complicated problems including medical diagnoses, finding the best alternative solution for a company for example; the best worker, the best job etc., logistic industry and so on. A relation has been developed between soft set and decision making method and it has overcome several problems[12, 13, 14, 15].

A lot of ideals were introduced over fuzzy sets. Mandal [16] introduced fuzzy ideals and definition of fuzzy interior ideals of ordered semirings. Dib and Galhum [17] defined fuzzy grupoid, a fuzzy semigroup and also fuzzy ideals and fuzzy bi-ideals of a semigroup. Moreover, Kazanciand Yamak [18] gave a kind of generalized fuzzy bi-ideals of semigroups. Kavikumar and Khamis [19] studied fuzzy ideals and fuzzy quasi ideals in ternary semirings. Then, Changphas and Summagrap [20] worked semigroups in covered ideals. Sezer and others wrote several papers [21, 22] over soft ideals in semigroups. In one of papers, they introduced soft union semigroup, ideals and bi-ideals [21]. After then, they defined soft intersection quasi ideals, generalized bi- ideals of a semigroup and surveyed regular, weakly regular, intra regular, completely regular, quasi regular semigroups with help of these ideals [22].

Fabrici $[23,24]$ investigated covered left, right ideal of a semigroup. By inspiring covered ideals, anti-covered (AC)-left (right), interior ideals were defined in [25] and Xie and Yan [25] obtained fuzzy anti-covered (AC)-left (right), bi-ideal, interior ideal of a semigroup and studied their basic properties. In [26], soft anti covered (AC)-ideals of semigroups were defined and studied in detailed.

In this manuscript, we first give the basic definitions of soft sets and covered ideals in semigroups. By using these basic definitions, we introduce covered ideals of semigroups by defining soft covered semigroups, soft covered left (right) ideals, soft covered bi-ideals, soft covered interior ideals, soft covered quasi-ideals, soft covered generalized bi-ideals. We discuss their properties and the interrelations with each others. Also, we study the relationship between soft anti covered ideals defined in [26] and soft covered ideals which is the most important point in this article.

\section{Methodology}

From now on, $\mathrm{U}$ refers to an initial universe, $\mathrm{E}$ is a set of parameters, $\mathrm{P}(\mathrm{U})$ is the power set of $U$ and $A, B, C \subseteq E$. 
Definition $1[1,13]$ A soft set $\mathrm{f}_{\mathrm{A}}$ over $\mathrm{U}$ is a set defined by

$$
\mathrm{f}_{\mathrm{A}}: \mathrm{E} \rightarrow \mathrm{P}(\mathrm{U}) \text { such thatf } \mathrm{A}(\mathrm{x})=\emptyset \text { if } \mathrm{x} \notin \mathrm{A} .
$$

Here $\mathrm{f}_{\mathrm{A}}$ is also called an approximate function. A soft set over $\mathrm{U}$ can be represented by the set of ordered pairs

$$
f_{A}=\left\{\left(x, f_{A}(x)\right): x \in E, f_{A}(x) \in P(U)\right\} .
$$

It is clear to see that a soft set is a parameterized family of subsets of the set $\mathrm{U}$. Note that the set of all soft sets over $\mathrm{U}$ will be denoted by $\mathrm{S}(\mathrm{U})$.

Definition 2 [13] Let $\mathrm{f}_{A}, \mathrm{f}_{\mathrm{B}} \in \mathrm{S}(\mathrm{U})$. Then, $\mathrm{f}_{\mathrm{A}}$ is called a soft subset of $\mathrm{f}_{\mathrm{B}}$ and denoted by $\mathrm{f}_{\mathrm{A}} \tilde{\subseteq} \mathrm{f}_{\mathrm{B}}$, if $\mathrm{f}_{\mathrm{A}}(\mathrm{x}) \subseteq \mathrm{f}_{\mathrm{B}}(\mathrm{x})$ for all $\mathrm{x} \in \mathrm{E}$.

Definition 3 [13] Let $\mathrm{f}_{A}, \mathrm{f}_{B} \in \mathrm{S}(\mathrm{U})$. Then, union of $\mathrm{f}_{\mathrm{A}}$ and $\mathrm{f}_{B}$, denoted by $\mathrm{f}_{A} \widetilde{\cup} \mathrm{f}_{B}$, is defined as $\mathrm{f}_{A} \widetilde{\cup}_{B}=f_{A \widetilde{B}}$, where $\mathrm{f}_{A \widetilde{\cup} \mathrm{B}}(\mathrm{x})=\mathrm{f}_{A}(\mathrm{x}) \cup \mathrm{f}_{B}(\mathrm{x})$, intersection of $\mathrm{f}_{A}$ and $\mathrm{f}_{B}$, denoted by $\mathrm{f}_{A} \widetilde{\cap} \mathrm{f}_{B}$, is defined as $\mathrm{f}_{A} \widetilde{\cap} \mathrm{f}_{B}=\mathrm{f}_{A \widetilde{\cap} \mathrm{B}}$, where $\mathrm{f}_{A \widetilde{\cap} \mathrm{B}}(\mathrm{x})=$ $\mathrm{f}_{\mathrm{A}}(\mathrm{x}) \cap \mathrm{f}_{\mathrm{B}}(\mathrm{x})$ for all $\mathrm{x} \in \mathrm{E}$.

Definition 4 [13] Let $\mathrm{f}_{A}, \mathrm{f}_{\mathrm{B}} \in \mathrm{S}(\mathrm{U})$. Then, $\widetilde{\wedge}$-product of $\mathrm{f}_{\mathrm{A}}$ and $\mathrm{f}_{\mathrm{B}}$, denoted

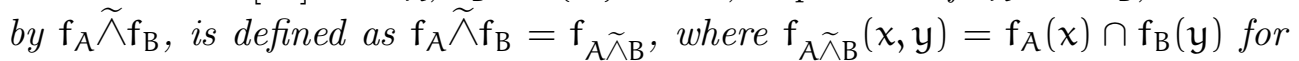
all $(x, y) \in \mathrm{E} \times \mathrm{E}$.

Definition 5 [27] Let $\mathrm{f}_{\mathrm{A}}$ and $\mathrm{f}_{\mathrm{B}}$ be soft sets over the common universe $\mathrm{U}$ and $\Psi$ be a function from $\mathrm{A}$ to $\mathrm{B}$. Then, soft image of $\mathrm{f}_{\mathrm{A}}$ under $\Psi$, denoted by $\Psi\left(f_{A}\right)$, is a soft set over $\mathrm{U}$ by

$$
\left(\Psi\left(f_{A}\right)\right)(b)=\left\{\begin{array}{lc}
\bigcap\left\{f_{A}(a) \mid a \in A \text { and } \Psi(a)=b\right\}, & \text { if } \Psi^{-1}(b) \neq \emptyset, \\
\emptyset, & \text { otherwise }
\end{array}\right.
$$

for all $\mathrm{b} \in \mathrm{B}$. And soft pre-image (or soft inverse image) of $\mathrm{f}_{\mathrm{B}}$ under $\Psi$, denoted by $\Psi^{-1}\left(f_{B}\right)$, is a soft set over $\mathrm{U}$ by $\left(\Psi^{-1}\left(f_{B}\right)\right)(a)=f_{B}(\Psi(a))$ for all $a \in A$.

From now on, $\mathrm{S}$ denotes a semigroup.

Definition 6 [21] Let $\mathrm{f}_{\mathrm{S}}$ and $\mathrm{g}_{\mathrm{S}}$ be soft sets over the common universe $\mathrm{U}$. Then, soft union product $\mathrm{f}_{\mathrm{S}} * \mathrm{~g}_{\mathrm{S}}$ is defined by

$$
\left(f_{S} * g_{S}\right)(x)= \begin{cases}\bigcap_{x=y z}\left\{f_{S}(y) \cup g_{S}(z)\right\}, & \text { if } \exists y, z \in S \text { such that } x=y z, \\ \emptyset, & \text { otherwise }\end{cases}
$$

for all $\mathrm{x} \in \mathrm{S}$. 
Theorem 1 [21] Let $\mathrm{f}_{\mathrm{S}}, \mathrm{g}_{\mathrm{S}}, \mathrm{h}_{\mathrm{S}} \in \mathrm{S}(\mathrm{U})$. Then,

i) $\left(f_{S} * g_{S}\right) * h_{S}=f_{S} *\left(g_{S} * h_{S}\right)$.

ii) $\mathrm{f}_{\mathrm{S}} * \mathrm{~g}_{\mathrm{S}} \neq \mathrm{g}_{\mathrm{S}} * \mathrm{f}_{\mathrm{S}}$, generally.

iii) $f_{S} *\left(g_{S} \widetilde{\cup} h_{S}\right)=\left(f_{S} * g_{S}\right) \widetilde{\cup}\left(f_{S} * h_{S}\right)$ and $\left(f_{S} \widetilde{\cup} g_{S}\right) * h_{S}=\left(f_{S} * h_{S}\right) \widetilde{\cup}\left(g_{S} * h_{S}\right)$.

iv) $f_{S} *\left(g_{S} \widetilde{\cap} h_{S}\right)=\left(f_{S} * g_{S}\right) \widetilde{\cap}\left(f_{S} * h_{S}\right)$ and $\left(f_{S} \widetilde{\cap} g_{S}\right) * h_{S}=\left(f_{S} * h_{S}\right) \widetilde{\cap}\left(g_{S} * h_{S}\right)$.

v) If $\mathrm{f}_{\mathrm{S}} \tilde{\widetilde{\subseteq}} \mathrm{g}_{\mathrm{S}}$, then $\mathrm{f}_{\mathrm{S}} * \mathrm{~h}_{\mathrm{S}} \tilde{\subseteq} \mathrm{g}_{\mathrm{S}} * \mathrm{~h}_{\mathrm{S}}$ and $\mathrm{h}_{\mathrm{S}} * \mathrm{f}_{\mathrm{S}} \underline{\widetilde{\subseteq}} \mathrm{h}_{\mathrm{S}} * \mathrm{~g}_{\mathrm{S}}$.

vi) If $\mathrm{t}_{\mathrm{S}}, \mathrm{l}_{\mathrm{S}} \in \mathrm{S}(\mathrm{U})$ such that $\mathrm{t}_{\mathrm{S}} \tilde{\subseteq} \mathrm{f}_{\mathrm{S}}$ and $\mathrm{l}_{\mathrm{S}} \tilde{\subseteq} \mathrm{g}_{\mathrm{S}}$, then $\mathrm{t}_{\mathrm{S}} * \mathrm{l}_{\mathrm{S}} \tilde{\widetilde{\subseteq}} \mathrm{f}_{\mathrm{S}} * \mathrm{~g}_{\mathrm{S}}$.

From now on, if $f_{S}: S \rightarrow P(U)$ is a soft set satisfying $f_{S}(x)=\emptyset$ for all $x \in S$, then $f_{S}$ is denoted by $\theta$ and if $f_{S}: S \rightarrow P(U)$ is a soft set satisfying $f_{S}(x)=U$ for all $x \in S$, then $f_{S}$ is denoted by $\mathbb{S}$.

Lemma 1 Let $\mathrm{f}_{\mathrm{S}}$ be any soft set over $\mathrm{U}$. Then, we have the followings.

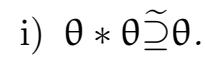

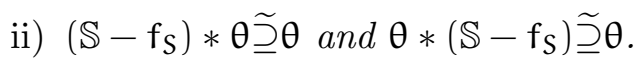

iii) $\left(\mathbb{S}-f_{S}\right) \widetilde{\cup} \theta=\left(\mathbb{S}-f_{S}\right)$ and $\left(\mathbb{S}-f_{S}\right) \widetilde{n} \theta=\theta$.

Soft anti covered (AC)-ideals of a semigroup were defined in [26] as following:

Definition 7 [26] $\mathrm{f}_{\mathrm{S}}$ is called as soft AC-semigroup over $\mathrm{U}$, if

$$
f_{S}(x y) \supseteq\left(\mathbb{S}-f_{S}\right)(x) \cap\left(\mathbb{S}-f_{S}\right)(y)
$$

for all $x, y \in S$.

Definition $8[26] \mathrm{f}_{\mathrm{S}}$ is called a soft $A C$-left ideal over $\mathrm{U}$, if $\mathrm{f}_{\mathrm{S}}(\mathrm{xy}) \supseteq(\mathbb{S}-$ $\left.\mathrm{f}_{\mathrm{S}}\right)(\mathrm{y}), A C$-right ideal of $\mathrm{S}$ over $\mathrm{U}$, if $\mathrm{f}_{\mathrm{S}}(\mathrm{xy}) \supseteq\left(\mathbb{S}-\mathrm{f}_{\mathrm{S}}\right)(\mathrm{x}), A C$-ideal of $\mathrm{S}$ over $\mathrm{U}$, if $\mathrm{f}_{\mathrm{S}}(\mathrm{xy}) \supseteq\left(\mathbb{S}-\mathrm{f}_{\mathrm{S}}\right)(\mathrm{y})$ and $\mathrm{f}_{\mathrm{S}}(\mathrm{xy}) \supseteq\left(\mathbb{S}-\mathrm{f}_{\mathrm{S}}\right)(\mathrm{x})$ for all $\mathrm{x}, \mathrm{y} \in \mathrm{S}$.

Definition 9 [26] A soft AC-semigroup $\mathrm{f}_{\mathrm{S}}$ over $\mathrm{U}$ is called a soft $A C$-bi-ideal over $\mathrm{U}$, if

$$
f_{S}(x y z) \supseteq\left(\mathbb{S}-f_{S}\right)(x) \cap\left(\mathbb{S}-f_{S}\right)(z)
$$


Definition 10 [26] $\mathrm{f}_{\mathrm{S}}$ is called a soft AC-interior ideal over $\mathrm{U}$, if

$$
f_{S}(x y z) \supseteq\left(\mathbb{S}-f_{S}\right)(y)
$$

and is called soft AC-generalized bi-ideal of $\mathrm{S}$, if

$$
f_{S}(x y z) \supseteq\left(\mathbb{S}-f_{S}\right)(x) \cap\left(\mathbb{S}-f_{S}\right)(y)
$$

for all $x, y, z \in S$.

From now on, all covered ideals are denoted by C-ideals for the sake of brevity. C-ideals of a semigroup (and fuzzy C-ideals of a semigroup) are defined in $[23,24]$ as following:

$\mathrm{L}$ is called C-left ideal of $\mathrm{S}$, if $\mathrm{L} \subseteq \mathrm{S}(\mathrm{S}-\mathrm{L})$ and $\mathrm{R}$ is called C-right ideal of $\mathrm{S}$, if $\mathrm{R} \subseteq(\mathrm{S}-\mathrm{R}) \mathrm{S}$. By $C$-two-sided ideal, it is meant a subset of $\mathrm{S}$, which is both C-left and C-right ideal of $S$. $X$ is called a $C$-interior of $S$ if $X \subseteq(S-X) S(S-X)$. $X$ is called C-bi-ideal of $S$ if $X \subseteq S(S-X) S$ and $X$ is called C-quasi-ideal of $S$ if $X \subseteq(S-X) S \cup S(S-X)$.

\section{Results and discussion}

\subsection{Soft C-semigroup}

In this section, we construct soft covered semigroup and study some properties of it.

Definition 11 Let $\mathrm{S}$ be semigroup and $\mathrm{f}_{\mathrm{S}}$ be soft set over $\mathrm{U} . \mathrm{f}_{\mathrm{S}}$ is called a soft covered semigroup, if

$$
f_{S}(x y) \subseteq\left(\mathbb{S}-f_{S}\right)(x) \cup\left(\mathbb{S}-f_{S}\right)(y)
$$

for all $x, y \in S$.

From now on, soft covered semigroup is denoted by soft C-semigroup for the sake of brevity.

Example 1 Consider the semigroup $\mathrm{S}=\{\mathrm{a}, \mathrm{b}, \mathrm{c}, \mathrm{d}\}$ constructed by the following table:

\begin{tabular}{c|cccc}
$\cdot$ & $a$ & $b$ & $c$ & $d$ \\
\hline$a$ & $a$ & $a$ & $a$ & $a$ \\
$b$ & $a$ & $a$ & $a$ & $a$ \\
$c$ & $a$ & $a$ & $b$ & $a$ \\
$d$ & $a$ & $a$ & $b$ & $b$
\end{tabular}


Let $\mathrm{U}=\mathrm{D}_{3}=\left\{\langle x, y\rangle: x^{3}=y^{2}=e, x y=y x^{2}\right\}=\left\{e, x, x^{2}, y, y x, y x^{2}\right\}$ be the universal set and $\mathrm{f}_{\mathrm{S}}$ be a soft set over $\mathrm{U}$ such that $\mathrm{f}_{\mathrm{S}}(\mathrm{a})=\emptyset, \mathrm{f}_{\mathrm{S}}(\mathrm{b})=$ $\{y\}, f_{S}(c)=\left\{e, y x^{2}\right\}, f_{S}(d)=\left\{e, x^{2}, y x, y x^{2}\right\}$ and so $\left(\mathbb{S}-f_{S}\right)(a)=\left\{e, x, x^{2}, y, y x\right.$, $\left.y x^{2}\right\},\left(\mathbb{S}-f_{S}\right)(b)=\left\{e, x, x^{2}, y x, y x^{2}\right\},\left(\mathbb{S}-f_{S}\right)(c)=\left\{x, x^{2}, y, y x\right\},\left(\mathbb{S}-f_{S}\right)(d)=$ $\{\mathrm{x}, \mathrm{y}\}$. One can show that $\mathrm{f}_{\mathrm{S}}$ is a soft C-semigroup. However, if $\mathrm{f}_{\mathrm{S}}(\mathrm{b})=$ $\left\{\mathrm{x}, \mathrm{x}^{2}, \mathrm{yx}\right\}$, we can easily show that $\mathrm{f}_{\mathrm{S}}$ is not a soft $C$-semigroup.

It is easy to see that if $\mathrm{f}_{\mathrm{S}}(\mathrm{x})=\emptyset$ for $\mathrm{x} \in \mathrm{S}$, then $\mathrm{f}_{\mathrm{S}}$ is a soft C-semigroup over $\mathrm{U}$. We denote such kind of C-semigroup by $\theta$.

Definition 12 [21] Let $\mathrm{X}$ be a subset of $\mathrm{S}$. We denote by $\mathcal{S}_{\mathrm{X}}^{\mathrm{c}}$ the soft anti characteristic function of $\mathrm{X}$ and define as

$$
\mathcal{S}_{X}^{\mathrm{c}}(\mathrm{x})= \begin{cases}\emptyset, & x \in X \\ \mathrm{U}, & x \in \mathcal{S}-\mathrm{X}\end{cases}
$$

It is clear that soft anti characteristic function is a soft set over U clearly,

$$
\mathcal{S}_{\mathrm{X}}^{\mathrm{c}}: \mathrm{S} \rightarrow \mathrm{P}(\mathrm{U}) \text {. }
$$

Theorem 2 If $\mathrm{X}$ is a $C$-semigroup of $\mathrm{S}$, then $\mathcal{S}_{\mathrm{X}}^{\mathrm{c}}$ is a soft $C$-semigroup of $\mathrm{S}$.

Proof. Let $X$ be a C-semigroup and $x=p q \in X$. Since $X \subseteq(S-X)(S-X)$, then it follows that $x=p q \in(S-X)(S-X)$, and so $p, q \in(S-X)$. In this statement, $\mathcal{S}_{X}^{\mathrm{C}}(\mathrm{pq}) \subseteq\left(\mathbb{S}-\mathcal{S}_{\mathrm{X}}^{\mathrm{c}}\right)(\mathrm{p}) \cup\left(\mathbb{S}-\mathcal{S}_{\mathrm{X}}^{\mathrm{c}}\right)(\mathrm{q})$, that is, $\mathcal{S}_{\mathrm{X}}^{\mathrm{c}}$ is a soft C-semigroup of $\mathrm{S}$. In fact,

$$
\begin{aligned}
\emptyset & =\mathcal{S}_{X}^{\mathrm{c}}(p q) \\
& \subseteq\left(\mathbb{S}-\mathcal{S}_{X}^{\mathrm{c}}\right)(\mathrm{p}) \cup\left(\mathbb{S}-\mathcal{S}_{X}^{\mathrm{c}}\right)(\mathrm{q}) \\
& =(\mathrm{U}-\mathrm{U}) \cup(\mathrm{U}-\mathrm{U}) \\
& =\emptyset .
\end{aligned}
$$

Theorem 3 Let $\mathrm{f}_{\mathrm{S}}$ be a soft set over $\mathrm{U}$. Then, $\mathrm{f}_{\mathrm{S}}$ is a soft AC-semigroup over $\mathrm{U}$ of $\mathrm{S}$ if and only if $\mathrm{f}_{\mathrm{S}}^{c}$ is a soft $C$-semigroup over $\mathrm{U}$ of $\mathrm{S}$.

Proof. Let $f_{S}$ be a soft AC-semigroup over $U$ of $S$. In this statement,

$$
\begin{aligned}
f_{S}^{c}(x y) & =\left(u-f_{S}\right)(x y) \\
& \subseteq U-\left(\left(\mathbb{S}-f_{S}\right)(x) \cap\left(\mathbb{S}-f_{S}\right)(y)\right) \\
& =\left(U-\left(\mathbb{S}-f_{S}\right)(x)\right) \cup\left(U-\left(\mathbb{S}-f_{S}\right)(y)\right) \\
& =\left(U-\left(U-f_{S}(x)\right) \cup\left(U-\left(U-f_{S}(y)\right)\right.\right. \\
& =\left(U-f_{S}^{c}(x)\right) \cup\left(U-f_{S}^{c}(y)\right) \\
& =\left(\mathbb{S}-f_{S}^{c}\right)(x) \cup\left(\mathbb{S}-f_{S}^{c}\right)(y)
\end{aligned}
$$


for all $x, y \in S$. Conversely, let $f_{S}^{c}$ be a soft C-semigroup over $U$ of $S$. Then,

$$
\begin{aligned}
f_{S}(x y) & =\left(U-f_{S}^{c}\right)(x y) \\
& \supseteq U-\left(\left(\mathbb{S}-f_{S}^{c}\right)(x) \cup\left(\mathbb{S}-f_{S}^{c}\right)(y)\right) \\
& \supseteq\left(U-\left(\mathbb{S}-f_{S}^{c}\right)(x)\right) \cap\left(U-\left(\mathbb{S}-f_{S}^{c}\right)(y)\right) \\
& =\left(U-\left(U-f_{S}^{c}(x)\right) \cap\left(U-\left(U-f_{S}^{c}(y)\right)\right.\right. \\
& =\left(U-f_{S}(x)\right) \cap\left(U-f_{S}(y)\right) \\
& \left.\left.=\left(\mathbb{S}-f_{S}\right)(x)\right) \cap\left(\mathbb{S}-f_{S}\right)(y)\right)
\end{aligned}
$$

for all $x, y \in S$. This completes the proof.

Theorem 4 Let $\mathrm{f}_{\mathrm{S}}$ be a soft set over $\mathrm{U}$. Then, $\mathrm{f}_{\mathrm{S}}$ is a soft $C$-semigroup over $\mathrm{U}$ if and only if we have:

$$
\mathrm{f}_{\mathrm{S}} \widetilde{\subseteq}\left(\mathbb{S}-\mathrm{f}_{\mathrm{S}}\right) *\left(\mathbb{S}-\mathrm{f}_{\mathrm{S}}\right)
$$

Proof. To prove this, we assume that $f_{S}$ is a soft C-semigroup over $\mathrm{U}$. If $f_{S}=\emptyset$, then it is trivial since

$$
\mathrm{f}_{\mathrm{S}}(\mathrm{x}) \subseteq\left(\left(\mathbb{S}-\mathrm{f}_{\mathrm{S}}\right) *\left(\mathbb{S}-\mathrm{f}_{\mathrm{S}}\right)\right)(\mathrm{x})
$$

thus, $f_{S} \subseteq\left(\mathbb{S}-f_{S}\right) *\left(\mathbb{S}-f_{S}\right)$. Otherwise, there exist elements $m, n \in S$ such that $x=m n$. Then, since $f_{S}$ is a soft $C$-semigroup over $U$, we have:

$$
\begin{aligned}
\left(\left(\mathbb{S}-f_{S}\right) *\left(\mathbb{S}-f_{S}\right)\right)(x) & =\bigcap_{x=m n}\left(\mathbb{S}-f_{S}\right)(m) \cup\left(\mathbb{S}-f_{S}\right)(n) \\
& \supseteq \bigcap_{x=m n} f_{S}(m n) \\
& =f_{S}(x)
\end{aligned}
$$

thus, $\mathrm{f}_{\mathrm{S}} \widetilde{\subseteq}\left(\mathbb{S}-\mathrm{f}_{\mathrm{S}}\right) *\left(\mathbb{S}-\mathrm{f}_{\mathrm{S}}\right)$.

Conversely, suppose that $f_{S} \widetilde{\subseteq}\left(\mathbb{S}-f_{S}\right) *\left(\mathbb{S}-f_{S}\right)$. Let $m, n \in S$ and $x=m n$. Hence, we have:

$$
\begin{aligned}
f_{S}(m n) & =f_{S}(x) \\
& \subseteq\left(\left(\mathbb{S}-f_{S}\right) *\left(\mathbb{S}-f_{S}\right)\right)(x) \\
& =\bigcap_{x=m n}\left(\mathbb{S}-f_{S}\right)(m) \cup\left(\mathbb{S}-f_{S}\right)(n) \\
& \subseteq\left(\mathbb{S}-f_{S}\right)(m) \cup\left(\mathbb{S}-f_{S}\right)(n) .
\end{aligned}
$$

Then, this means that $f_{S}$ is a soft $\mathrm{C}$-semigroup over $\mathrm{U}$. This completes the proof.

Proposition 1 Let $\mathrm{f}_{\mathrm{S}}$ and $\mathrm{f}_{\mathrm{T}}$ be soft $C$-semigroups over $\mathrm{U}$. Then, $\mathrm{f}_{\mathrm{S}} \widetilde{\wedge} \mathrm{f}_{\mathrm{T}}$ is a soft $C$-semigroup over $\mathrm{U}$. 
Proof. Let $\left(x_{1}, y_{1}\right),\left(x_{2}, y_{2}\right)$ be any two elements of $S \times T$. Then,

$$
\begin{aligned}
\mathrm{f}_{\mathrm{S} \tilde{} \sim \mathrm{T}}\left(\left(x_{1}, y_{1}\right),\left(x_{2}, y_{2}\right)\right)= & f_{\mathrm{S} \wedge T}\left(x_{1} x_{2}, y_{1} y_{2}\right) \\
= & f_{S}\left(x_{1} x_{2}\right) \cap f_{T}\left(y_{1} y_{2}\right) \\
\subseteq & {\left[\left(\mathbb{S}-f_{S}\right)\left(x_{1}\right) \cup\left(\mathbb{S}-f_{S}\right)\left(x_{2}\right)\right] \cap\left[\left(\mathbb{S}-f_{T}\right)\left(y_{1}\right)\right.} \\
& \left.\cup\left(\mathbb{S}-f_{T}\right)\left(y_{2}\right)\right] \\
= & {\left[U-\left(f_{S}\left(x_{1}\right) \cap f_{S}\left(x_{2}\right)\right)\right] \cap\left[\left(U-\left(f_{T}\left(y_{1}\right) \cap f_{T}\left(y_{2}\right)\right)\right]\right.} \\
= & U-\left[\left(f_{S}\left(x_{1}\right) \cap f_{S}\left(x_{2}\right)\right) \cup\left(f_{T}\left(y_{1}\right) \cap f_{T}\left(y_{2}\right)\right)\right] \\
\subseteq & U-\left[\left(f_{S}\left(x_{1}\right) \cap f_{S}\left(x_{2}\right)\right) \cap\left(f_{T}\left(y_{1}\right) \cap f_{T}\left(y_{2}\right)\right)\right] \\
= & U-\left[\left(f_{S}\left(x_{1}\right) \cap f_{T}\left(y_{1}\right)\right) \cap\left(f_{S}\left(x_{2}\right) \cap f_{T}\left(y_{2}\right)\right)\right] \\
= & \left(S-f_{S} \tilde{A T}_{T}\right)\left(x_{1}, y_{1}\right) \cup\left(\mathbb{S}-f_{S} \tilde{S}_{T}\right)\left(x_{2}, y_{2}\right) .
\end{aligned}
$$

This implies that $f_{S} \widetilde{\wedge} f_{T}$ is a soft C-semigroup over $U$.

Theorem 5 Let $\mathrm{f}_{\mathrm{S}}$ and $\mathrm{g}_{\mathrm{S}}$ be two soft $C$-semigroups over $\mathrm{U}$, then $\mathrm{f}_{\mathrm{S}} \widetilde{\cap} \mathrm{g}_{\mathrm{S}}$ is also so.

Proof. Let $f_{S}$ and $g_{S}$ be two soft C-semigroups over $\mathrm{U}$ for $x, y \in S$. Then,

$$
\begin{aligned}
\left(f_{S} \widetilde{\cap} g_{S}\right)(x y) & =f_{S}(x y) \cap g_{S}(x y) \\
& \subseteq\left[\left(\mathbb{S}-f_{S}\right)(x) \cup\left(\mathbb{S}-f_{S}\right)(y)\right] \cap\left[\left(\mathbb{S}-g_{S}\right)(x) \cup\left(\mathbb{S}-g_{S}\right)(y)\right] \\
& =\left[u-\left(f_{S}(x) \cap f_{S}(y)\right)\right] \cap\left[u-\left(g_{S}(x) \cap g_{S}(y)\right)\right] \\
& =u-\left[\left(f_{S}(x) \cap f_{S}(y)\right) \cup\left(g_{S}(x) \cap g_{S}(y)\right)\right] \\
& \subseteq u-\left[\left(f_{S}(x) \cap f_{S}(y) \cap\left(g_{S}(x) \cap g_{S}(y)\right)\right]\right. \\
& =u-\left[( f _ { S } ( x ) \cap g _ { S } ( x ) ) \cap \left(\left(f_{S}(y) \cap g_{S}(y)\right]\right.\right. \\
& =u-\left[\left(f_{S} \widetilde{\cap} g_{S}\right)(x) \cap\left(f_{S} \widetilde{\cap} g_{S}\right)(y)\right] \\
& =\left(\mathbb{S}-\left(f_{S} \widetilde{\cap} g_{S}\right)\right)(x) \cup\left(\mathbb{S}-\left(f_{S} \widetilde{\cap} g_{S}\right)\right)(y) .
\end{aligned}
$$

This completes the proof.

The union of two C-semigroups needs not to be a soft C-semigroup as shown in following example.

Example 2 Let $\mathrm{f}_{\mathrm{S}}$ and $\mathrm{g}_{\mathrm{S}}$ be two soft sets over $\mathrm{U}=\mathrm{D}_{3}$ of semigroup $\mathrm{S}=$ $\{\mathrm{a}, \mathrm{b}, \mathrm{c}, \mathrm{d}\}$. We accept that $\mathrm{f}_{\mathrm{S}}$ is the same soft set in Example 1 and define the soft set, $g_{S}$ as following; $g_{S}(a)=\emptyset, g_{S}(b)=\{e, x\}, g_{S}(c)=\left\{x^{2}, y x, y x^{2}\right\}$ and $g_{S}(d)=\left\{y, y x, y x^{2}\right\}$. Since $\left(f_{S} \widetilde{\cup} g_{S}\right)(d d)=\left(f_{S} \widetilde{\cup} g_{S}\right)(b) \nsubseteq\left(\mathbb{S}-\left(f_{S} \widetilde{\cup} g_{S}\right)\right)(d) \cup$ $\left(\mathbb{S}-\left(f_{S} \widetilde{\cup} g_{S}\right)\right)(d), f_{S} \widetilde{\cup} g_{S}$ is not a soft C-semigroup over $\mathrm{U}$.

Proposition 2 Let $\mathrm{f}_{\mathrm{S}}$ and $\mathrm{f}_{\mathrm{T}}$ be soft $C$-semigroups over $\mathrm{U}$ and $\Psi$ is a semigroup isomorphism from $\mathrm{S}$ to $\mathrm{T}$. If $\mathrm{f}_{\mathrm{S}}$ is a soft C-semigroup over $\mathrm{U}$, then $\Psi\left(\mathrm{f}_{\mathrm{S}}\right)$ is a soft C-semigroup. 
Proof. Let $m_{1}, m_{2} \in T$. Since $\Psi$ is surjective, then there exist $k_{1}, k_{2} \in S$ such that $\Psi\left(k_{1}\right)=m_{1}$ and $\Psi\left(k_{2}\right)=m_{2}$. Then,

$$
\begin{aligned}
\left(\Psi\left(f_{S}\right)\right)\left(m_{1} m_{2}\right)= & \bigcap\left\{f_{S}(k): k \in S, \Psi(k)=m_{1} m_{2}\right\} \\
= & \bigcap\left\{f_{S}(k): k \in S, k=\Psi^{-1}\left(m_{1} m_{2}\right)\right\} \\
= & \bigcap\left\{f_{S}(k): k \in S, k=\Psi^{-1}\left(\Psi\left(k_{1} k_{2}\right)\right)=k_{1} k_{2}\right\} \\
= & \bigcap\left\{f_{S}\left(k_{1} k_{2}\right): k_{i} \in S, \Psi\left(k_{i}\right)=m_{i}, i=1,2\right\} \\
\subseteq & \left.\bigcap\left(\mathbb{S}-f_{S}\right)\left(k_{1}\right) \cup\left(\mathbb{S}-f_{S}\right)\left(k_{2}\right): m_{i} \in S, \Psi\left(k_{i}\right)=m_{i}, i=1,2\right\} \\
= & \left(\bigcap\left\{\left(\mathbb{S}-f_{S}\right)\left(k_{1}\right): k_{1} \in S, \Psi\left(k_{1}\right)=m_{1}\right\}\right) \\
& \cup\left(\bigcap\left\{\left(\mathbb{S}-f_{S}\right)\left(k_{2}\right): k_{2} \in S, \Psi\left(k_{2}\right)=m_{2}\right\}\right) \\
\subseteq & \left(\bigcup\left\{\left(\mathbb{S}-f_{S}\right)\left(k_{1}\right): k_{1} \in S, \Psi\left(k_{1}\right)=m_{1}\right\}\right) \\
& \cup\left(\bigcup\left\{\left(\mathbb{S}-f_{S}\right)\left(k_{2}\right): k_{2} \in S, \Psi\left(k_{2}\right)=m_{2}\right\}\right) \\
= & \left\{U-\bigcap\left(f_{S}\left(k_{1}\right)\right): k_{1} \in S, \Psi\left(k_{1}\right)=m_{1}\right\} \\
& \cup\left\{U-\bigcap\left(f_{S}\left(k_{2}\right)\right): k_{2} \in S, \Psi\left(k_{2}\right)=m_{2}\right\} \\
= & \left(\mathbb{S}-\Psi\left(f_{S}\right)\right)\left(m_{1}\right) \cup\left(\mathbb{S}-\Psi\left(f_{S}\right)\right)\left(m_{2}\right) .
\end{aligned}
$$

Consequently, $\Psi\left(f_{S}\right)$ is a soft C-semigroup over $\mathrm{U}$.

Proposition 3 Let $\mathrm{f}_{\mathrm{S}}$ and $\mathrm{f}_{\mathrm{T}}$ be soft $C$-semigroups over $\mathrm{U}$ and $\Psi$ be a semigroup homomorphism from $\mathrm{S}$ to $\mathrm{T}$. If $\mathrm{f}_{\mathrm{T}}$ is a soft $C$-semigroup over $\mathrm{U}$, then so $\Psi^{-1}\left(f_{\mathrm{T}}\right)$ is.

Proof. Suppose $k_{1}, k_{2} \in S$. Then,

$$
\begin{aligned}
\left(\Psi^{-1}\left(f_{\mathrm{T}}\right)\right)\left(k_{1} k_{2}\right) & =f_{\mathrm{T}}\left(\Psi\left(k_{1} k_{2}\right)\right. \\
& =f_{\mathrm{T}}\left(\Psi\left(k_{1}\right) \Psi\left(k_{2}\right)\right) \\
& \subseteq\left(\mathbb{S}-f_{\mathrm{T}}\right)\left(\Psi\left(k_{1}\right)\right) \cup\left(\mathbb{S}-f_{\mathrm{T}}\right)\left(\Psi\left(k_{2}\right)\right) \\
& =\mathrm{U}-\left(\mathrm { f } _ { \mathrm { T } } \left(\Psi\left(\mathrm{k}_{1}\right) \cap \mathrm{f}_{\mathrm{T}}\left(\Psi\left(\mathrm{k}_{2}\right)\right)\right.\right. \\
& =\mathrm{U}-\left(\Psi^{-1}\left(\mathrm{f}_{\mathrm{T}}\left(\mathrm{k}_{1}\right)\right) \cap \Psi^{-1}\left(\mathrm{f}_{\mathrm{T}}\left(\mathrm{k}_{2}\right)\right)\right) \\
& =\left(\mathbb{S}-\Psi^{-1}\left(\mathrm{f}_{\mathrm{T}}\right)\right)\left(\mathrm{k}_{1}\right) \cup\left(\mathbb{S}-\Psi^{-1}\left(\mathrm{f}_{\mathrm{T}}\right)\right)\left(\mathrm{k}_{2}\right) .
\end{aligned}
$$

Hence, $\Psi^{-1}\left(f_{T}\right)$ is a soft $C$-semigroup over $U$.

\subsection{Soft C-left (right), C-ideals of semigroups}

In this subsection, soft covered left (right) ideal are introduced and also we survey some properties of these ideals.

Definition 13 Let $\mathrm{S}$ be semigroup and $\mathrm{f}_{\mathrm{S}}$ be soft set over $\mathrm{U} . \mathrm{f}_{\mathrm{S}}$ is called a soft covered left ideal over $\mathrm{U}$ if $\mathrm{f}_{\mathrm{S}}(\mathrm{xy}) \subseteq\left(\mathbb{S}-\mathrm{f}_{\mathrm{S}}\right)(\mathrm{y})$; covered right ideal of $\mathrm{S}$ over $\mathrm{U}$ if $\mathrm{f}_{\mathrm{S}}(\mathrm{xy}) \subseteq\left(\mathbb{S}-\mathrm{f}_{\mathrm{S}}\right)(\mathrm{x})$; covered ideal of $\mathrm{S}$ over $\mathrm{U}$ if $\mathrm{f}_{\mathrm{S}}(\mathrm{xy}) \subseteq\left(\mathbb{S}-\mathrm{f}_{\mathrm{S}}\right)(\mathrm{y})$ and $\mathrm{f}_{\mathrm{S}}(\mathrm{x} \mathrm{y}) \subseteq\left(\mathbb{S}-\mathrm{f}_{\mathrm{S}}\right)(\mathrm{x})$ for all $\mathrm{x}, \mathrm{y} \in \mathrm{S}$. 
From now on, soft covered left ideal is denoted by soft C-left ideal, soft covered right ideal by soft C-right ideal and soft covered ideal by soft C-ideal for the sake of brevity.

Example 3 Let $\mathrm{S}=\{\mathrm{a}, \mathrm{b}, \mathrm{c}, \mathrm{d}\}$ in Example 1. Define the soft set $\mathrm{f}_{\mathrm{S}}$ over $\mathrm{U}=\mathrm{D}_{4}$ as following, $\mathrm{f}_{\mathrm{S}}(\mathrm{a})=\emptyset, \quad \mathrm{f}_{\mathrm{S}}(\mathrm{b})=\{x, y\}, \quad \mathrm{f}_{\mathrm{S}}(\mathrm{c})=\left\{e, x^{2}, \mathrm{y} x^{2}\right\}$, $f_{S}(d)=\left\{x^{2}, y x, y x^{2}\right\}$ and so $\left(\mathbb{S}-f_{S}\right)(a)=\left\{e, x, x^{2}, y, y x, y x^{2}\right\}, \quad\left(\mathbb{S}-f_{S}\right)(b)=$ $\left\{e, x^{2}, y x, y x^{2}\right\}, \quad\left(\mathbb{S}-f_{S}\right)(c)=\{x, y, y x\}, \quad\left(\mathbb{S}-f_{S}\right)(d)=\{e, x, y\}$. Then, $f_{S}$ forms a soft C-left ideal of $\mathrm{S}$ over $\mathrm{U}$ but now suppose $\mathrm{f}_{\mathrm{S}}(\mathrm{b})=\left\{\mathrm{x}, \mathrm{x}^{2}, \mathrm{yx}, \mathrm{y} \mathrm{x}^{2}\right\}$, then since $\mathrm{f}_{\mathrm{S}}(\mathrm{b})=\mathrm{f}_{\mathrm{S}}(\mathrm{dd}) \nsubseteq\left(\mathbb{S}-\mathrm{f}_{\mathrm{S}}\right)(\mathrm{d}), \mathrm{f}_{\mathrm{S}}$ is not a soft C-left ideal.

It is easy to see that if $f_{S}(x)=\emptyset$ for $x \in S$, then $f_{S}$ is a soft C-left (right) ideal over $\mathrm{U}$. We denote such kind of C-left (right) by $\theta$.

Theorem 6 If $\mathrm{X}$ is a C-left ideal of $\mathrm{S}$, then $\mathcal{S}_{\mathrm{X}}^{\mathrm{c}}$ is a soft C-left ideal of $\mathrm{S}$.

Proof. Assume that $X$ is a C-left ideal and $x=m n \in X$. Then, since $X \subseteq$ $S(S-X), x=m n \in S(S-X)$, implying that $m \in S$ and $n \in S-X$. Hence, $\mathcal{S}_{X}^{\mathrm{c}}(\mathrm{mn}) \subseteq\left(\mathbb{S}-\mathcal{S}_{X}^{\mathrm{c}}\right)(\mathrm{n})$. In fact,

$$
\emptyset=\mathcal{S}_{X}^{\mathrm{c}}(\mathrm{mn}) \subseteq\left(\mathbb{S}-\mathcal{S}_{X}^{\mathrm{c}}\right)(\mathrm{n})=\mathrm{U}-\mathrm{U}=\emptyset .
$$

Thus, $\mathcal{S}_{X}^{\mathrm{c}}$ is a soft C-left ideal of $\mathrm{S}$.

Theorem 7 Let $\mathrm{f}_{\mathrm{S}}$ be a soft set over $\mathrm{U}$. Then, $\mathrm{f}_{\mathrm{S}}$ is a soft AC-left (right, ideal) over $\mathrm{U}$ of $\mathrm{S}$ if and only if $\mathrm{f}_{\mathrm{S}}^{\mathrm{c}}$ is a soft C-left (right, ideal) ideal over $\mathrm{U}$ of $\mathrm{S}$.

Proof. We give the proof for soft AC-left ideals. Let $f_{S}$ be a soft AC-left ideal over $\mathrm{U}$ of S. In this statement,

$$
\begin{aligned}
f_{S}^{c}(x y) & =\left(U-f_{S}\right)(x y) \\
& \subseteq U-\left(\mathbb{S}-f_{S}\right)(y) \\
& \subseteq U-\left(U-f_{S}(y)\right) \\
& =\left(U-f_{S}^{c}(y)\right) \\
& =\left(\mathbb{S}-f_{S}^{c}\right)(y)
\end{aligned}
$$

for all $x, y \in S$. Conversely, let $f_{S}^{c}$ be a soft C-left ideal over $U$ of $S$. Then,

$$
\begin{aligned}
f_{S}(x y) & =\left(U-f_{S}^{c}\right)(x y) \\
& \supseteq U-\left(\left(\mathbb{S}-f_{S}^{c}\right)\right)(y) \\
\supseteq & U-\left(U-f_{S}^{c}(y)\right) \\
& =\left(U-f_{S}(y)\right) \\
& =\left(\mathbb{S}-f_{S}\right)(y)
\end{aligned}
$$


for all $x, y \in S$. This completes the proof.

Theorem 8 Let $\mathrm{f}_{\mathrm{S}}$ be soft set over $\mathrm{U}$. Then, $\mathrm{f}_{\mathrm{S}}$ is a soft C-left ideal over $\mathrm{U}$ if and only if

$$
f_{S} \widetilde{\subseteq} \theta *\left(\mathbb{S}-f_{S}\right)
$$

Proof. Assume that $\mathrm{f}_{\mathrm{S}}$ is a soft C-left ideal over $\mathrm{U}$. In this statement, if $\mathrm{f}_{\mathrm{S}}=\emptyset$, it is clear that $f_{S} \widetilde{\subseteq}\left(\theta *\left(\mathbb{S}-f_{S}\right)\right)$. According to assume, since $f_{S}$ is a soft C-left ideal for $m, n \in S$ over $\mathrm{U}$, we have:

$$
\begin{aligned}
\left(\theta *\left(\mathbb{S}-f_{S}\right)\right)(m n) & =\bigcap_{s=m n} \theta(m) \cup\left(\mathbb{S}-f_{S}\right)(n) \\
& \supseteq \bigcap_{s=m n} \emptyset \cup\left(f_{S}\right)(m n) \\
& =\bigcap_{s=m n}\left(f_{S}\right)(m n) \\
& =f_{S}(s) \\
& =f_{S}(m n)
\end{aligned}
$$

hence, $f_{S} \widetilde{\subseteq} \theta *\left(\mathbb{S}-f_{S}\right)$. Conversely, suppose that $f_{S} \widetilde{\subseteq}\left(\theta *\left(\mathbb{S}-f_{S}\right)\right)$. Let $m, n \in S$ and $s=m n$. Then, we have:

$$
\begin{aligned}
\left(f_{S}\right)(m n) & =\left(f_{S}\right)(s) \\
& \subseteq\left(\theta *\left(\mathbb{S}-f_{S}\right)\right)(m n) \\
& =\bigcap_{m n=x y} \theta(x) \cup\left(\mathbb{S}-f_{S}\right)(y) \\
& \subseteq \emptyset \cup\left(\mathbb{S}-f_{S}\right)(n) \\
& =\left(\mathbb{S}-f_{S}\right)(n) .
\end{aligned}
$$

Theorem 9 Let $\mathrm{f}_{\mathrm{S}}$ be soft set over $\mathrm{U}$. Then, $\mathrm{f}_{\mathrm{S}}$ is a soft $C$-right ideal over $\mathrm{U}$ if and only if

$$
\mathrm{f}_{\mathrm{S}} \widetilde{\widetilde{\subseteq}}\left(\mathbb{S}-\mathrm{f}_{\mathrm{S}}\right) * \theta .
$$

Theorem 10 Let $\mathrm{f}_{\mathrm{S}}$ be soft set over $\mathrm{U}$. Then, $\mathrm{f}_{\mathrm{S}}$ is a soft $C$-ideal over $\mathrm{U}$ if and only if $\mathrm{f}_{\mathrm{S}} \widetilde{\subseteq} \theta *\left(\mathbb{S}-\mathrm{f}_{\mathrm{S}}\right)$ and $\mathrm{f}_{\mathrm{S}} \widetilde{\subseteq}\left(\mathbb{S}-\mathrm{f}_{\mathrm{S}}\right) * \theta$.

Theorem 11 Let $\mathrm{f}_{\mathrm{S}}$ and $\mathrm{g}_{\mathrm{S}}$ be two soft subsets of $\mathrm{S}$ such that $\mathrm{g}_{\mathrm{S}} \widetilde{\widetilde{\subseteq}} \mathrm{f}_{\mathrm{S}}$. If $\mathrm{f}_{\mathrm{S}}$ is a soft C-left ideal, then $\mathrm{g}_{\mathrm{S}}$ is also a soft C-left ideal.

Proof. First assume that $f_{S}$ is soft C-left ideal and $g_{S} \widetilde{\subseteq} f_{S}$. Let $p, q \in S$ and 
$s=p q$. Then,

$$
\begin{aligned}
g_{S}(p q) \subseteq f_{S}(p q) & \subseteq\left(\theta *\left(\mathbb{S}-f_{S}\right)\right)(p q) \\
& =\bigcap_{s=p q} \theta(p) \cup\left(\mathbb{S}-f_{S}\right)(q) \\
& =\bigcap_{s=p q} \emptyset \cup\left(\mathbb{S}-f_{S}\right)(q) \\
& =\bigcap_{s=p q}\left(\mathbb{S}-f_{S}\right)(q) \\
& \subseteq \bigcap_{s=p q}\left(\mathbb{S}-g_{S}\right)(q) \\
& =\bigcap_{s=p q} \theta(p) \cup\left(\mathbb{S}-g_{S}\right)(q) \\
& =\left(\theta *\left(\mathbb{S}-g_{S}\right)\right)(p q) .
\end{aligned}
$$

Consequently, $g_{S}(p q) \subseteq\left(\theta *\left(\mathbb{S}-g_{S}\right)\right)(p q)$ meaning that $g_{S}$ is a soft C-left ideal.

Definition 14 Soft union left and soft C-left ideal $\mathrm{f}_{\mathrm{S}}$ of $\mathrm{S}$ is called a completely soft C-left ideal of S.

Theorem 12 Let $\mathbf{f}_{\mathrm{S}}$ be a soft subset of $\mathrm{S}$. Then, the followings are equivalent.

i) $\mathrm{f}_{\mathrm{S}}$ is a completely soft C-left ideal of $\mathrm{S}$.

ii) $(\forall x, y \in S) f_{S}(x y) \subseteq f_{S}(y) \cap\left(\mathbb{S}-f_{S}\right)(y)$.

iii) $\left(\forall s \in S^{2}\right) f_{S}(s) \subseteq \bigcap_{s=x y} f_{S}(y) \cap \bigcap_{s=x y}\left(\mathbb{S}-f_{S}\right)(y)$.

\section{Proof.}

$\mathrm{i} \Rightarrow$ ii Suppose that $f_{S}$ is a completely soft C-left ideal of $S$. Then, since $f_{S}$ is a soft C-left ideal $f_{S}(x y) \subseteq\left(\mathbb{S}-f_{S}\right)(y)$. Since $f_{S}$ is a soft union left ideal of $S, f_{S}(x y) \subseteq f_{S}(y)$. This means that $f_{S}(x y) \subseteq f_{S}(y) \cap\left(\mathbb{S}-f_{S}\right)(y)$.

ii $\Rightarrow$ iii Accept that $(\forall x, y \in S) f_{S}(x y) \subseteq f_{S}(y) \cap\left(\mathbb{S}-f_{S}\right)(y)$. Then, by taking into account the sets, $f_{S}(x y) \subseteq f_{S}(y)$ and $f_{S}(x y) \subseteq\left(\mathbb{S}-f_{S}\right)(y)$. Let $s=x y$ for any $x, y \in S$. This implies that $f_{S}(s)=f_{S}(x y) \subseteq \bigcap_{s=x y} f_{S}(y) \cap \bigcap_{s=x y}\left(\mathbb{S}-f_{S}\right)(y)$.

iii $\Rightarrow$ i Assume that $\forall s=x y \in S^{2}, f_{S}(s) \subseteq \bigcap_{s=x y} f_{S}(y) \cap \bigcap_{x=y z}\left(\mathbb{S}-f_{S}\right)(y)$. Thus, $f_{S}(x y) \subseteq \bigcap_{s=x y} f_{S}(y)$ and $f_{S}(x y) \subseteq \bigcap_{s=x y}\left(\mathbb{S}-f_{S}\right)(y)$. Then,

$$
\begin{aligned}
f_{S}(s)=f_{S}(x y) & \subseteq \bigcap_{s=x y}\left(\mathbb{S}-f_{S}\right)(y) \\
& =\bigcap_{s=x y} \emptyset \cup\left(\mathbb{S}-f_{S}\right)(y) \\
& =\bigcap_{s=x y} \theta(x) \cup\left(\mathbb{S}-f_{S}\right)(y) \\
& =\left(\theta *\left(\mathbb{S}-f_{S}\right)\right)(s)
\end{aligned}
$$

and also,

$$
\begin{aligned}
f_{S}(x y) & \subseteq \bigcap_{s=x y} f_{S}(y) \\
& \subseteq f_{S}(y) .
\end{aligned}
$$

Clearly, $f_{S}$ is a completely soft C-left ideal of $S$. 
Theorem 13 If $\mathrm{f}_{\mathrm{S}}$ and $\mathrm{g}_{\mathrm{S}}$ are two soft $C$-left ideals of $\mathrm{S}$ over $\mathrm{U}$, then $\mathrm{f}_{\mathrm{S}} \widetilde{\cap} \mathrm{g}_{\mathrm{S}}$ is a soft C-left ideal over $\mathrm{U}$.

Proof. Assume that $f_{S}$ and $g_{S}$ are two soft C-left ideals. Then,

$$
\begin{aligned}
\left(f_{S} \widetilde{\cap} g_{S}\right)(m n) & =f_{S}(m n) \cap g_{S}(m n) \\
& \subseteq\left(\mathbb{S}-f_{S}\right)(n) \cap\left(\mathbb{S}-g_{S}\right)(n) \\
& =u-\left(f_{S}(n) \cup g_{S}(n)\right) \\
& \subseteq u-\left(f_{S}(n) \cap g_{S}(n)\right) \\
& =\left(\mathbb{S}-\left(f_{S} \widetilde{\cap} g_{S}\right)\right)(n)
\end{aligned}
$$

for all $m, n \in S$. This means that $f_{S} \widetilde{\cap} g_{S}$ is a soft C-left ideal over $U$.

Now, we show that if $f_{S}$ and $g_{S}$ are two soft C-left ideals of $S$ over $U$, then $f_{S} \widetilde{\cup} g_{S}$ is not a soft C-left ideal of $S$ with the following example.

Example 4 Let consider the semigroup $\mathrm{S}=\{\mathrm{a}, \mathrm{b}, \mathrm{c}, \mathrm{d}\}$ over $\mathrm{U}=\mathrm{D}_{4}$ in Example 1 and introduce the soft set $\mathrm{f}_{\mathrm{S}}$ over $\mathrm{U}$ as following $\mathrm{f}_{\mathrm{S}}(\mathrm{a})=\emptyset$, $\mathrm{f}_{\mathrm{S}}(\mathrm{b})=\{e, x\}, \quad \mathrm{f}_{\mathrm{S}}(\mathrm{c})=\left\{\mathrm{x}^{2}, \mathrm{yx}, \mathrm{yx^{2 }}\right\}, \quad \mathrm{f}_{\mathrm{S}}(\mathrm{d})=\left\{\mathrm{y}, \mathrm{x}^{2}, \mathrm{y} \mathrm{x}^{2}\right\}$ so $\left(\mathbb{S}-\mathrm{f}_{\mathrm{S}}\right)(\mathrm{a})=$ $\left\{e, x, x^{2}, y, y x, y x^{2}\right\}, \quad\left(\mathbb{S}-f_{S}\right)(b)=\left\{y, x^{2}, y x, y x^{2}\right\}, \quad\left(\mathbb{S}-f_{S}\right)(c)=\{e, x, y\}, \quad(\mathbb{S}-$ $\left.\mathrm{f}_{\mathrm{S}}\right)(\mathrm{d})=\{e, x, y x\}$. Also let $\mathrm{g}_{\mathrm{S}}(\mathrm{a})=\emptyset, \quad \mathrm{g}_{\mathrm{S}}(\mathrm{b})=\{e\}, \mathrm{g}_{\mathrm{S}}(\mathrm{c})=\left\{\mathrm{y}, \mathrm{x}^{2}, \mathrm{yx}, \mathrm{y} \mathrm{x}^{2}\right\}$, $g_{S}(d)=\left\{x, x^{2}, y x, y x^{2}\right\}$ so $\left(\mathbb{S}-g_{S}\right)(a)=\left\{e, x, x^{2}, y, y x, y x^{2}\right\}, \quad\left(\mathbb{S}-g_{S}\right)(b)=$ $\left\{x, y, x^{2}, y x^{2}\right\}, \quad\left(\mathbb{S}-g_{S}\right)(c)=\{e, x\}, \quad\left(\mathbb{S}-g_{S}\right)(d)=\{e, y\}$. This shows that $\left(f_{S} \widetilde{\cup} g_{S}\right)(d d)=\left(f_{S} \widetilde{\cup} g_{S}\right)(b) \nsubseteq\left(\mathbb{S}-\left(f_{S} \widetilde{\cup} g_{S}\right)\right)(d)$.

Proposition $4 \mathrm{f}_{\mathrm{S}}$ is a soft C-ideal over $\mathrm{U}$ of $\mathrm{S}$ if and only if

$$
f_{S}(m n) \subseteq\left(\mathbb{S}-f_{S}\right)(m) \cap\left(\mathbb{S}-f_{S}\right)(n)
$$

for all $\mathrm{m}, \mathrm{n} \in \mathrm{S}$.

Proof. Let $f_{S}$ be a soft C-ideal of $S$ over $U$ and $m, n \in S$. Then, $f_{S}(m n) \subseteq$ $\left(\mathbb{S}-f_{S}\right)(m)$ and $f_{S}(m n) \subseteq\left(\mathbb{S}-f_{S}\right)(n)$. Then, $f_{S}(m n) \subseteq\left(\mathbb{S}-f_{S}\right)(m) \cap\left(\mathbb{S}-f_{S}\right)(n)$.

Conversely, suppose that $f_{S}(m n) \subseteq\left(\mathbb{S}-f_{S}\right)(m) \cap\left(\mathbb{S}-f_{S}\right)(n)$ for all $m, n \in S$. It follows that

$$
f_{S}(m n) \subseteq\left(\mathbb{S}-f_{S}\right)(m) \cap\left(\mathbb{S}-f_{S}\right)(n) \subseteq\left(\mathbb{S}-f_{S}\right)(m)
$$

and

$$
f_{S}(m n) \subseteq\left(\mathbb{S}-f_{S}\right)(m) \cap\left(\mathbb{S}-f_{S}\right)(n) \subseteq\left(\mathbb{S}-f_{S}\right)(n)
$$

so $f_{S}$ is a soft $\mathrm{C}$-ideal of $\mathrm{S}$ over $\mathrm{U}$. 
Theorem 14 Let $\mathrm{f}_{\mathrm{S}}$ be soft set over $\mathrm{U}$. If $\mathrm{f}_{\mathrm{S}}$ is a soft C-left (right) ideal over $\mathrm{U}, \mathrm{f}_{\mathrm{S}}$ is a soft C-semigroup over $\mathrm{U}$.

Proof. We give the proof for soft C-left ideals. Similarly, it can be indicated for soft C-right ideals. Let $f_{S}$ be a soft C-left ideal of $S$ over $U$. Then, $f_{S}(p q) \subseteq$ $\left(\mathbb{S}-f_{S}\right)(q)$ for all $p, q \in S$. Thus, $f_{S}(p q) \subseteq\left(\mathbb{S}-f_{S}\right)(q) \subseteq\left(\mathbb{S}-f_{S}\right)(p) \cup\left(\mathbb{S}-f_{S}\right)(q)$, therefore $f_{S}$ is a soft C-semigroup.

Proposition 5 Let $\mathrm{f}_{\mathrm{S}}$ be soft set over $\mathrm{U}$. Then, $\left(\mathbb{S}-\mathrm{f}_{\mathrm{S}}\right) \widetilde{\cap}\left(\theta *\left(\mathbb{S}-\mathrm{f}_{\mathrm{S}}\right)\right)$ is a soft $C$-left ideal over $\mathrm{U}$ and $\left(\mathbb{S}-\mathrm{f}_{\mathrm{S}}\right) \widetilde{\cap}\left(\left(\mathbb{S}-\mathrm{f}_{\mathrm{S}}\right) * \theta\right)$ is a soft $C$-right ideal of $\mathrm{S}$ over $\mathrm{U}$.

Proof. Assume that $f_{S}$ is a soft C-left ideal of $S$. Then,

$$
\begin{aligned}
\theta *\left[\left(\left(\mathbb{S}-f_{S}\right) \widetilde{\cap}\left(\theta *\left(\mathbb{S}-f_{S}\right)\right)\right)\right] & =\left[\left(\theta *\left(\mathbb{S}-f_{S}\right)\right)\right] \widetilde{\cap}\left[\left(\theta *\left(\theta *\left(\mathbb{S}-f_{S}\right)\right)\right]\right. \\
& =\left(\theta *\left(\mathbb{S}-f_{S}\right)\right) \widetilde{\cap}\left((\theta * \theta) *\left(\mathbb{S}-f_{S}\right)\right) \\
& \supseteq\left(\theta *\left(\mathbb{S}-f_{S}\right)\right) \widetilde{\cap}\left(\left(\theta *\left(\mathbb{S}-f_{S}\right)\right)\right. \\
& =\left(\theta *\left(\mathbb{S}-f_{S}\right)\right) \\
& \simeq\left(\mathbb{S}-f_{S}\right) \widetilde{\cap}\left(\theta *\left(\mathbb{S}-f_{S}\right)\right) .
\end{aligned}
$$

Clearly, $\left(\mathbb{S}-f_{S}\right) \widetilde{\cap}\left(\theta *\left(\mathbb{S}-f_{S}\right)\right)$ is a soft C-left ideal of $S$ over $U$. Also,

$$
\begin{aligned}
{\left[\left(\left(\mathbb{S}-f_{S}\right) \widetilde{\cap}\left(\left(\mathbb{S}-f_{S}\right) * \theta\right)\right)\right] * \theta } & =\left[\left(\left(\mathbb{S}-f_{S}\right) * \theta\right)\right] \widetilde{\cap}\left[\left(\left(\left(\mathbb{S}-f_{S}\right) * \theta\right) * \theta\right)\right] \\
& =\left(\left(\mathbb{S}-f_{S}\right) * \theta\right) \widetilde{\cap}\left(\left(\left(\mathbb{S}-f_{S}\right) *(\theta * \theta)\right)\right. \\
& \supseteq\left(\left(\mathbb{S}-f_{S}\right) * \theta\right) \widetilde{\cap}\left(\left(\mathbb{S}-f_{S}\right) * \theta\right) \\
& =\left(\left(\mathbb{S}-f_{S}\right) * \theta\right) \\
& \left.\supseteq \widetilde{D}-f_{S}\right) \widetilde{\cap}\left(\left(\mathbb{S}-f_{S}\right) * \theta\right) .
\end{aligned}
$$

Hence, $\left(\mathbb{S}-f_{S}\right) \widetilde{\cap}\left(\left(\mathbb{S}-f_{S}\right) * \theta\right)$ is a soft C-right ideal of $\mathbf{S}$ over $\mathrm{U}$.

Theorem 15 Let $\mathrm{f}_{\mathrm{S}}$ and $\mathrm{g}_{\mathrm{S}}$ be a soft $C$-right ideal and soft $C$-left ideal of $\mathrm{S}$ over $\mathrm{U}$, respectively. Then,

$$
f_{S} \widetilde{\cup} g_{S} \widetilde{\subseteq}\left(\mathbb{S}-f_{S}\right) *\left(\mathbb{S}-g_{S}\right) .
$$

Proof. We know that $f_{S}$ is a soft C-R-right ideal of $S$ over $U$ and $g_{S}$ is a soft C-left ideal of $S$ over $U$ and also $\theta \widetilde{\subseteq}\left(\mathbb{S}-f_{S}\right), \theta \widetilde{\subseteq}\left(\mathbb{S}-g_{S}\right)$. Thus,

$$
g_{S} \widetilde{\subseteq} \theta *\left(\mathbb{S}-g_{S}\right) \widetilde{\subseteq}\left(\mathbb{S}-f_{S}\right) *\left(\mathbb{S}-g_{S}\right)
$$

and

$$
f_{S} \widetilde{\subseteq}\left(\mathbb{S}-f_{S}\right) * \theta \widetilde{\subseteq}\left(\mathbb{S}-f_{S}\right) *\left(\mathbb{S}-g_{S}\right)
$$


from here

$$
f_{S} \widetilde{\cup} g_{S} \widetilde{\subseteq}\left(\mathbb{S}-f_{S}\right) *\left(\mathbb{S}-g_{S}\right) .
$$

Now, we survey that again, let $f_{S}$ and $g_{S}$ be a soft C-right ideal a soft C-left ideal of $\mathrm{S}$ over $\mathrm{U}$, respectively. In this statement,

$$
\mathrm{f}_{S} \widetilde{\cap} \mathrm{g}_{S} \underset{\nsupseteq}{ }\left(\mathbb{S}-\mathrm{f}_{S}\right) *\left(\mathbb{S}-\mathrm{g}_{\mathrm{S}}\right) .
$$

with the following example.

Example 5 Think the semigroup $\mathrm{S}=\{\mathrm{a}, \mathrm{b}, \mathrm{c}, \mathrm{d}\}$ over $\mathrm{U}=\mathrm{D}_{4}$ in Example 1 and let the soft set $\mathrm{f}_{\mathrm{S}}$ and $\mathrm{gS}_{\mathrm{S}}$ be $\mathrm{f}_{\mathrm{S}}(\mathrm{b})=\{\mathrm{x}, \mathrm{yx}\},\left(\mathbb{S}-\mathrm{f}_{\mathrm{S}}\right)(\mathrm{c})=\{\mathrm{e}, \mathrm{x}, \mathrm{yx}\}$, $\left(\mathbb{S}-f_{S}\right)(d)=\{x, y, y x\}$ and $g_{S}(b)=\left\{y x, y x^{2}\right\},\left(\mathbb{S}-g_{S}\right)(c)=\left\{x, y x, y x^{2}\right\}$, $\left(\mathbb{S}-\mathrm{g}_{\mathrm{S}}\right)(\mathrm{d})=\left\{\mathrm{yx}, \mathrm{yx}^{2}\right\}$. Then, $\mathrm{f}_{\mathrm{S}}$ is a soft C-right ideal and $\mathrm{g}_{\mathrm{S}}$ is a soft C-left ideal.

$$
\begin{aligned}
\left(\left(\mathbb{S}-f_{S}\right) *\left(\mathbb{S}-g_{S}\right)\right)(b) & =\left\{\left(\mathbb{S}-f_{S}\right)(d) \cup\left(\mathbb{S}-g_{S}\right)(d)\right\} \cap\left\{\left(\mathbb{S}-f_{S}\right)(c) \cup\left(\mathbb{S}-g_{S}\right)(c)\right\} \\
& \cap\left\{\left(\mathbb{S}-f_{S}\right)(d) \cup\left(\mathbb{S}-g_{S}\right)(c)\right\} \\
& =\left\{x, y x, y x^{2}\right\} \\
& \nsubseteq \subseteq\left(f_{S} \cap g_{S}\right)(b) \\
& =\{y x\} .
\end{aligned}
$$

Proposition 6 Let $\mathrm{f}_{\mathrm{S}}$ and $\mathrm{f}_{\mathrm{T}}$ be soft C-left (right) ideals of $\mathrm{S}$ over $\mathrm{U}$. Again, $\mathrm{f}_{\mathrm{S}} \widetilde{\sim} \mathrm{f}_{\mathrm{T}}$ is a soft C-left (right) ideal of $\mathrm{S} \times \mathrm{T}$ over $\mathrm{U}$.

Proof. We accept that $f_{S}$ and $f_{T}$ are soft C-left ideals of $S$ over $U$ and $\left(x_{1}, y_{1}\right),\left(x_{2}, y_{2}\right) \in S \times T$,

$$
\begin{aligned}
\mathrm{f}_{\mathrm{S} \tilde{\top} \mathrm{T}}\left(\left(x_{1}, y_{1}\right),\left(x_{2}, y_{2}\right)\right) & =f_{S \tilde{S}_{T}}\left(x_{1} x_{2}, y_{1} y_{2}\right) \\
& =f_{S}\left(x_{1} x_{2}\right) \cap f_{T}\left(y_{1} y_{2}\right) \\
& \subseteq\left(\mathbb{S}-f_{S}\right)\left(x_{2}\right) \cap\left(\mathbb{S}-f_{T}\right)\left(y_{2}\right) \\
& =U-\left(f_{S}\left(x_{2}\right) \cup f_{T}\left(y_{2}\right)\right) \\
& \subseteq U-\left(f_{S}\left(x_{2}\right) \cap f_{T}\left(y_{2}\right)\right) \\
& =\left(S-f_{S} \tilde{S T}_{T}\right)\left(x_{2}, y_{2}\right) .
\end{aligned}
$$

Therefore, $\mathrm{f}_{\mathrm{S}} \widetilde{\sim}_{\mathrm{T}}$ is a soft C-left ideal over $\mathrm{U}$.

We give following propositions without proof. The proofs are similar to those in section 2 .

Proposition 7 Let $\mathrm{f}_{\mathrm{S}}$ and $\mathrm{h}_{\mathrm{S}}$ be soft sets over $\mathrm{U}$ and $\Psi$ is a semigroup isomorphism from $\mathrm{S}$ to $\mathrm{T}$. If $\mathrm{f}_{\mathrm{S}}$ is a soft C-left (right) ideal of $\mathrm{S}$ over $\mathrm{U}$, then so is $\Psi\left(\mathrm{f}_{\mathrm{S}}\right)$ of $\mathrm{T}$ over $\mathrm{U}$. 
Proposition 8 Let $\mathrm{f}_{\mathrm{S}}$ and $\mathrm{h}_{\mathrm{S}}$ be soft sets over $\mathrm{U}$ and $\Psi$ is a semigroup homomorphism from $\mathrm{S}$ to $\mathrm{T}$. If $\mathrm{f}_{\mathrm{T}}$ is a soft C-left (right) ideal of $\mathrm{T}$ over $\mathrm{U}$, then so is $\Psi^{-1}\left(f_{\mathrm{T}}\right)$ of $\mathrm{S}$ over $\mathrm{U}$.

\subsection{Soft C-bi-ideals of semigroups}

In this subsection, we define soft covered bi-ideals and provide their basic properties by using soft set operations and soft intersection products and also support them with examples.

Definition 15 A soft $C$-semigroup $\mathrm{f}_{\mathrm{S}}$ over $\mathrm{U}$ is called a soft covered bi-ideal over $\mathrm{U}$ if

$$
f_{S}(x y z) \subseteq\left(\mathbb{S}-f_{S}\right)(x) \cup\left(\mathbb{S}-f_{S}\right)(z) .
$$

For the sake of brevity, soft covered bi-ideal is denoted by soft C-bi-ideal.

Example 6 Define operation over $S=\mathbb{Z}_{4}=\{0,1,2,3\}$ by the following table:

\begin{tabular}{l|llll}
. & 0 & 1 & 2 & 3 \\
\hline 0 & 0 & 0 & 0 & 0 \\
1 & 0 & 0 & 0 & 0 \\
2 & 0 & 0 & 0 & 1 \\
3 & 0 & 0 & 1 & 2
\end{tabular}

Now let $\mathrm{U}=\mathrm{D}_{2}=\{e, x, y, y x\}$ be universal set and $\mathrm{f}_{\mathrm{S}}$ be a soft set over $\mathrm{U}$ defined by $\mathrm{f}_{\mathrm{S}}(0)=\emptyset, \mathrm{f}_{\mathrm{S}}(1)=\{x\}, \mathrm{f}_{\mathrm{S}}(2)=\{x, y\}, \mathrm{f}_{\mathrm{S}}(3)=\{\mathrm{yx}\}$ and so $\left(\mathbb{S}-f_{S}\right)(0)=U,\left(\mathbb{S}-f_{S}\right)(1)=\{e, y, y x\}, \quad\left(\mathbb{S}-f_{S}\right)(2)=\{e, y x\}, \quad\left(\mathbb{S}-f_{S}\right)(3)=$ $\{\boldsymbol{e}, \boldsymbol{x}, \mathrm{y}\}$. Then, $\mathrm{f}_{\mathrm{S}}$ is a soft $C$-bi-ideal, but if $\mathrm{f}_{\mathrm{S}}(\mathbf{1})=\{\boldsymbol{e}, \mathrm{yx}\}$, then $\mathrm{f}_{\mathrm{S}}$ is not a soft C-bi-ideal.

It is easy to see that if $f_{S}(x)=\emptyset$ for $x \in S$, then $f_{S}$ is a soft C-bi-ideal over $U$. We denote such kind of C-bi-ideal by $\theta$.

Theorem 16 If $\mathrm{X}$ is a $C$-bi-ideal of $\mathrm{S}$, then $\mathcal{S}_{\mathrm{X}}$ is a soft $C$-bi-ideal of $\mathrm{S}$.

Proof. We accept that $X$ is a C-bi-ideal of $S$. Let $x=m n p \in X$, then it is clear that $x=m n p \in(S-X) S(S-X)$, implying that $m, p \in S-X$ and $n \in S$. In this statement, $\left.\mathcal{S}_{X}(\mathrm{mnp}) \subseteq\left(\mathbb{S}-\mathcal{S}_{X}\right)(\mathrm{m})\right) \cup\left(\mathbb{S}-\mathcal{S}_{X}\right)(\mathrm{p})$. In fact,

$$
\begin{aligned}
\emptyset & =\mathcal{S}_{X}^{\mathrm{c}}(\mathrm{mnp}) \\
& \subseteq\left(\mathbb{S}-\mathcal{S}_{X}^{\mathrm{c}}\right)(\mathrm{m}) \cup\left(\mathbb{S}-\mathcal{S}_{\mathrm{X}}^{\mathrm{c}}\right)(\mathrm{p}) \\
& =(\mathrm{U}-\mathrm{U}) \cup(\mathrm{U}-\mathrm{U}) \\
& =\emptyset .
\end{aligned}
$$


Theorem 17 Let $\mathrm{f}_{\mathrm{S}}$ be a soft set over $\mathrm{U}$. Then, $\mathrm{f}_{\mathrm{S}}$ is a soft AC-bi-ideal over $\mathrm{U}$ of $\mathrm{S}$ if and only if $\mathrm{f}_{\mathrm{S}}^{\mathrm{c}}$ is a soft $C$-bi-ideal over $\mathrm{U}$ of $\mathrm{S}$.

Proof. Let $f_{S}$ be a soft AC-bi-ideal over $U$ of $S$. In this statement,

$$
\begin{aligned}
f_{S}^{\mathcal{c}}(x y z) & =\left(U-f_{S}\right)(x y z) \\
& \subseteq U-\left(\left(\mathbb{S}-f_{S}\right)(x) \cap\left(\mathbb{S}-f_{S}\right)(z)\right) \\
& =U-\left(\left(U-f_{S}(x)\right) \cap\left(U-f_{S}(z)\right)\right. \\
& =\left(U-\left(U-f_{S}(x)\right) \cup\left(U-\left(U-f_{S}(z)\right)\right.\right. \\
& =\left(U-f_{S}^{c}(x)\right) \cup\left(U-f_{S}^{c}(z)\right) \\
& =\left(\mathbb{S}-f_{S}^{c}\right)(x) \cup\left(\mathbb{S}-f_{S}^{c}\right)(z)
\end{aligned}
$$

for all $x, y, z \in S$. Conversely, let $f_{S}^{c}$ be a soft C-bi-ideal over $U$ of $S$. Then,

$$
\begin{aligned}
f_{S}(x y z) & =\left(U-f_{S}^{c}\right)(x y z) \\
\supseteq & U-\left(\left(\mathbb{S}-f_{S}^{c}\right)(x) \cup\left(\mathbb{S}-f_{S}^{c}\right)(z)\right) \\
& =\left(U-\left(U-f_{S}^{c}(x)\right) \cap\left(U-\left(U-f_{S}^{c}(z)\right)\right.\right. \\
& =\left(U-f_{S}(x)\right) \cap\left(U-f_{S}(z)\right) \\
& =\left(\mathbb{S}-f_{S}\right)(x) \cap\left(\mathbb{S}-f_{S}\right)(z)
\end{aligned}
$$

for all $x, y, z \in S$. This completes the proof.

Theorem 18 A soft subset $\mathrm{f}_{\mathrm{S}}$ of $\mathrm{S}$ is a soft $C$-bi-ideal if and only if

$$
\mathrm{f}_{\mathrm{S}} \widetilde{\simeq}\left(\mathbb{S}-\mathrm{f}_{\mathrm{S}}\right) * \theta *\left(\mathbb{S}-\mathrm{f}_{\mathrm{S}}\right)
$$

Proof. Let $f_{S}$ be a soft C-bi-ideal of $S$. Assume that $f_{S}=\emptyset$, then it is clear $\left.f_{S} \widetilde{\subseteq}\left(\left(\mathbb{S}-f_{S}\right) * \theta\right) *\left(\mathbb{S}-f_{S}\right)\right)$. Otherwise, let $a, b, t, p, z \in S$. Since $f_{S}$ is a soft C-bi-ideal of $\mathrm{S}$ over $\mathrm{U}$, then,

$$
\begin{aligned}
\left(\left(\mathbb{S}-f_{S}\right) * \theta *\left(\mathbb{S}-f_{S}\right)\right)(s) & =\bigcap_{s=a b}\left[\left(\left(\mathbb{S}-f_{S}\right) * \theta\right)(a) \cup\left(\mathbb{S}-f_{S}\right)(b)\right] \\
& =\bigcap_{s=a b}\left[\bigcap_{a=t p}\left(\mathbb{S}-f_{S}\right)(t) \cup \theta(p) \cup\left(\mathbb{S}-f_{S}\right)(b)\right] \\
& =\bigcap_{s=a b}\left[\bigcap_{a=t p}\left(\mathbb{S}-f_{S}\right)(t) \cup \emptyset \cup\left(\mathbb{S}-f_{S}\right)(b)\right] \\
& =\bigcap_{S=a b} \bigcap_{a=t p}\left(\mathbb{S}-f_{S}\right)(t) \cup\left(\mathbb{S}-f_{S}\right)(b) \\
& \supseteq \bigcap_{s=a b} \bigcap_{a=t p} f_{S}(t p b) \\
& =\bigcap_{s=a b} f_{S}(a b) \\
& =f_{S}(s)
\end{aligned}
$$

hence, $\mathrm{f}_{\mathrm{S}} \widetilde{\subseteq}\left(\left(\mathbb{S}-\mathrm{f}_{\mathrm{S}}\right) * \theta *\left(\mathbb{S}-\mathrm{f}_{\mathrm{S}}\right)\right)$. 
Conversely, let us assume that $\left.\mathrm{f}_{\mathrm{S}} \widetilde{\subseteq}\left(\left(\mathbb{S}-\mathrm{f}_{\mathrm{S}}\right) * \theta\right) *\left(\mathbb{S}-\mathrm{f}_{\mathrm{S}}\right)\right)$. Let $\mathrm{s}=\mathrm{abz} \in \mathrm{S}$,

$$
\begin{aligned}
& f_{S}(a b z)=f_{S}(s) \\
& \subseteq\left(\left(\mathbb{S}-\mathrm{f}_{\mathrm{S}}\right) * \theta *\left(\mathbb{S}-\mathrm{f}_{\mathrm{S}}\right)\right)(\mathrm{s}) \\
& \left.=\bigcap_{\mathrm{s}=\mathrm{ab} z=\mathrm{mn}}\left(\left(\mathbb{S}-\mathrm{f}_{\mathrm{S}}\right) * \theta\right)(\mathrm{m}) \cup\left(\mathbb{S}-\mathrm{f}_{\mathrm{S}}\right)(\mathrm{n})\right) \\
& \left.\subseteq\left(\left(\mathbb{S}-f_{S}\right) * \theta\right)(a b) \cup\left(\mathbb{S}-f_{S}\right)(z)\right) \\
& =\bigcap_{\mathrm{ab}=\mathrm{tp}}\left(\mathbb{S}-\mathrm{f}_{\mathrm{S}}\right)(\mathrm{t}) \cup \theta(\mathrm{p}) \cup\left(\mathbb{S}-\mathrm{f}_{\mathrm{S}}\right)(z) \\
& \subseteq\left(\mathbb{S}-f_{S}\right)(a) \cup \emptyset \cup\left(\mathbb{S}-f_{S}\right)(z) \\
& =\left(\mathbb{S}-f_{S}\right)(a) \cup\left(\mathbb{S}-f_{S}\right)(z) \text {. }
\end{aligned}
$$

Consequently, $f_{S}$ is a soft C-bi-ideal of $S$ over $U$. This completes the proof.

Theorem 19 The intersection of two soft $C$-bi-ideals over $\mathrm{U}$ is a soft $C$-biideal over $\mathrm{U}$.

Proof. Let $f_{S}$ and $g_{S}$ be soft C-bi-ideals over $\mathrm{U}$. Then,

$$
\begin{aligned}
\left(f_{S} \widetilde{\cap} g_{S}\right)(m n p) & =f_{S}(m n p) \cap g_{S}(m n p) \\
& \subseteq\left[\left(\mathbb{S}-f_{S}\right)(m) \cup\left(\mathbb{S}-f_{S}\right)(p)\right] \cap\left[\left(\mathbb{S}-g_{S}\right)(m) \cup\left(\mathbb{S}-g_{S}\right)(p)\right] \\
& =\left[U-\left(f_{S}(m) \cap f_{S}(p)\right)\right] \cap\left[U-\left(g_{S}(m) \cap g_{S}(p)\right)\right] \\
& =U-\left[\left(f_{S}(m) \cap f_{S}(p)\right) \cup\left(g_{S}(m) \cap g_{S}(p)\right)\right] \\
& \subseteq U-\left[\left(f_{S}(m) \cap f_{S}(p) \cap\left(g_{S}(m) \cap g_{S}(p)\right)\right]\right. \\
& =U-\left(( f _ { S } ( m ) \cap g _ { S } ( m ) ) \cap \left(\left(f_{S}(p) \cap g_{S}(p)\right)\right.\right. \\
& =U-\left(\left(f_{S} \widetilde{\cap} g_{S}\right)(m) \cap\left(f_{S} \widetilde{\cap} g_{S}\right)(p)\right) \\
& =\left(U-\left(f_{S} \widetilde{\cap} g_{S}\right)(m)\right) \cup\left(U-\left(f_{S} \widetilde{\cap} g_{S}\right)(p)\right) \\
& =\left(\mathbb{S}-\left(f_{S} \widetilde{\cap} g_{S}\right)\right)(m) \cup\left(\mathbb{S}-\left(f_{S} \widetilde{\cap} g_{S}\right)\right)(p)
\end{aligned}
$$

for $m, n, p \in S$. This completes the proof. Now, we show that if $f_{S}$ and $g_{S}$ are two C-bi-ideals of $S$ over $\mathrm{U}$, then $\mathrm{f}_{S} \widetilde{\cup} g_{S}$ is not a soft C-bi-ideal of $S$ with the following example.

Example 7 Consider the semigroup $\mathrm{S}=\mathrm{Z}_{4}=\{0,1,2,3\}$ and define the soft set $\mathrm{f}_{\mathrm{S}}$ and $\mathrm{g}_{\mathrm{S}}$ over $\mathrm{U}=\mathrm{D}_{2}$ in Example 6 as following, respectively. $\mathrm{f}_{\mathrm{S}}(0)=\emptyset$, $\mathrm{f}_{\mathrm{S}}(1)=\{x\}, \mathrm{f}_{\mathrm{S}}(2)=\{e\}, \mathrm{f}_{\mathrm{S}}(3)=\{\mathrm{y}, \mathrm{yx}\}$ so $\left(\mathbb{S}-\mathrm{f}_{\mathrm{S}}\right)(0)=\mathrm{U},\left(\mathbb{S}-\mathrm{f}_{\mathrm{S}}\right)(1)=$ $\{e, y, y x\},\left(\mathbb{S}-f_{S}\right)(2)=\{x, y, y x\},\left(\mathbb{S}-f_{S}\right)(3)=\{e, x\}$ and $g_{S}(0)=\emptyset, g_{S}(1)=\{e\}$, $g_{S}(2)=\{e\}, g_{S}(3)=\{x, y x\}$ so $\left(\mathbb{S}-g_{S}\right)(0)=U,\left(\mathbb{S}-g_{S}\right)(1)=\{x, y, y x\},(\mathbb{S}-$ $\left.\mathrm{g}_{\mathrm{S}}\right)(2)=\{\mathrm{x}, \mathrm{y}, \mathrm{yx}\},\left(\mathbb{S}-\mathrm{g}_{\mathrm{S}}\right)(3)=\{e, y\}$. Then, $\mathrm{f}_{\mathrm{S}}$ and $\mathrm{g}_{\mathrm{S}}$ are two $C$-bi-ideals of $\mathrm{S}$ over $\mathrm{U}$, since $\left(\mathrm{f}_{S} \widetilde{\cup} \mathrm{g}_{S}\right)(333)=\left(\mathrm{f}_{S} \widetilde{\cup} \mathrm{g}_{S}\right)(1) \nsubseteq\left(\mathbb{S}-\left(\mathrm{f}_{S} \widetilde{\cup} \mathrm{g}_{S}\right)(3)\right) \cup\left(\mathbb{S}-\left(\mathrm{f}_{S} \widetilde{\cup} \mathrm{g}_{S}\right)(3)\right)$, $\left(\mathrm{f}_{\mathrm{S}} \widetilde{\cup} \mathrm{g}_{\mathrm{S}}\right)$ is not a soft $C$-bi-ideal. 
Theorem 20 Every soft C-left (right) ideal of semigroup $\mathrm{S}$ over $\mathrm{U}$ is a soft C-bi-ideal of $\mathrm{S}$ over $\mathrm{U}$.

Proof. Assume that $f_{S}$ is a soft C-Left ideal of $S$ over $U$ for $m, p, q \in S$. Then,

$$
f_{S}(m p q)=f_{S}((m p) q) \subseteq\left(\mathbb{S}-f_{S}\right)(q) \subseteq\left(\mathbb{S}-f_{S}\right)(m) \cup\left(\mathbb{S}-f_{S}\right)(q) .
$$

Hence, $f_{S}$ is a soft $\mathrm{C}$-bi-ideal of $\mathrm{S}$.

Proposition 9 Let $\mathrm{f}_{\mathrm{S}}$ and $\mathrm{f}_{\mathrm{T}}$ be soft $C$-bi-ideals of $\mathrm{S}$ over $\mathrm{U}$. Then, $\mathrm{f}_{\mathrm{S}} \widetilde{ }_{\mathrm{f}_{\mathrm{T}}}$ is a soft $C$-bi-ideal of $\mathrm{S} \times \mathrm{T}$ over $\mathrm{U}$.

Proof. We know that $f_{S}$ and $f_{T}$ are soft C-bi-ideals and there exists $\left(x_{1}, y_{1}\right)$, $\left(x_{2}, y_{2}\right),\left(x_{3}, y_{3}\right) \in S \times T$,

$$
\begin{aligned}
f_{S \wedge T}\left(\left(x_{1}, y_{1}\right),\left(x_{2}, y_{2}\right),\left(x_{3}, y_{3}\right)\right) & \left.=f_{S \wedge T}\left(x_{1} x_{2} x_{3}, y_{1} y_{2} y_{3}\right)\right) \\
& =f_{S}\left(x_{1} x_{2} x_{3}\right) \cap f_{T}\left(y_{1} y_{2} y_{3}\right) \\
& \subseteq\left[\left(\mathbb{S}-f_{S}\right)\left(x_{1}\right) \cup\left(\mathbb{S}-f_{S}\right)\left(x_{3}\right)\right] \cap\left[\left(\mathbb{S}-f_{T}\right)\left(y_{1}\right)\right. \\
& \left.\cup\left(\mathbb{S}-f_{T}\right)\left(y_{3}\right)\right] \\
& =\left[U-\left(f_{S}\left(x_{1}\right) \cap f_{S}\left(x_{3}\right)\right)\right] \cap\left[\left(U-\left(f_{T}\left(y_{1}\right) \cap f_{T}\left(y_{3}\right)\right)\right]\right. \\
& =U-\left[\left(f_{S}\left(x_{1}\right) \cap f_{S}\left(x_{3}\right)\right) \cup\left(f_{T}\left(y_{1}\right) \cap f_{T}\left(y_{3}\right)\right)\right] \\
& \subseteq U-\left[\left(f_{S}\left(x_{1}\right) \cap f_{S}\left(x_{3}\right)\right) \cap\left(f_{T}\left(y_{1}\right) \cap f_{T}\left(y_{3}\right)\right)\right] \\
& =U-\left[\left(f_{S}\left(x_{1}\right) \cap f_{T}\left(x_{3}\right)\right) \cap\left(f_{S}\left(y_{1}\right) \cap f_{T}\left(y_{3}\right)\right)\right] \\
& =\left(\mathbb{S}-f_{S \wedge T}\right)\left(x_{1}, y_{1}\right) \cup\left(\mathbb{S}-f_{S \wedge} \tilde{s}_{T}\right)\left(x_{3}, y_{3}\right) .
\end{aligned}
$$

This shows that $f_{S} \widetilde{\wedge} f_{T}$ is a soft C-bi-ideal over $u$.

Proposition 10 Let $\mathrm{f}_{\mathrm{S}}$ and $\mathrm{h}_{\mathrm{S}}$ be soft sets over $\mathrm{U}$ and $\Psi$ is a semigroup isomorphism from $\mathrm{S}$ to $\mathrm{T}$. If $\mathrm{f}_{\mathrm{S}}$ is a soft $C$-bi-ideal of $\mathrm{S}$ over $\mathrm{U}$, then so is $\Psi\left(f_{\mathrm{S}}\right)$ of $\mathrm{T}$ over $\mathrm{U}$.

Proposition 11 Let $\mathrm{f}_{\mathrm{S}}$ and $\mathrm{h}_{\mathrm{S}}$ be soft sets over $\mathrm{U}$ and $\Psi$ is a semigroup homomorphism from $\mathrm{S}$ to $\mathrm{T}$. If $\mathrm{f}_{\mathrm{T}}$ is a soft $C$-bi-ideal of $\mathrm{T}$ over $\mathrm{U}$, then so is $\Psi^{-1}\left(f_{\mathrm{T}}\right)$ of $\mathrm{S}$ over $\mathrm{U}$.

\subsection{Soft C-interior ideal of semigroups}

In this section, we introduce soft covered interior ideals of semigroups, obtain their basic properties with respect to soft operations and soft intersection product. 
Definition 16 Let $\mathrm{f}_{\mathrm{S}}$ be soft set over $\mathrm{U}$ and $\mathrm{x}, \mathrm{y}, z \in \mathrm{S}$. If

$$
f_{S}(x y z) \subseteq\left(\mathbb{S}-f_{S}\right)(y)
$$

$\mathrm{f}_{\mathrm{S}}$ called a soft covered interior ideal over $\mathrm{U}$.

For the sake of brevity, soft covered interior ideal is denoted by soft C-interior ideal over $\mathrm{U}$.

Example 8 Let think the semigroup $\mathbb{Z}_{4}$ and the soft set $\mathrm{f}_{\mathrm{S}}$ over $\mathrm{U}=\mathrm{D}_{2}=$ $\{\mathrm{e}, \mathrm{x}, \mathrm{y}, \mathrm{yx}\}$ in Example 6 . Then, one can easily show that $\mathrm{f}_{\mathrm{S}}$ is a soft $C$-interior ideal of $\mathrm{S}$ over $\mathrm{U}$. But we accept that $\mathrm{f}_{\mathrm{S}}(\mathbf{1})=\{\mathrm{e}, \mathrm{y}\}$, then $\mathrm{f}_{\mathrm{S}}(333)=\mathrm{f}_{\mathrm{S}}(\mathbf{1}) \nsubseteq$ $\left(\mathbb{S}-\mathrm{f}_{\mathrm{S}}\right)(3)$ which implies $\mathrm{f}_{\mathrm{S}}$ is not a soft C-interior ideal.

It is easy to see that if $f_{S}(x)=\emptyset$ for $x \in S$, then $f_{S}$ is a soft C-interior-ideal over $\mathrm{U}$. We denote such kind of C-interior-ideal by $\theta$.

Theorem 21 If $\mathrm{X}$ is a C-interior ideal of $\mathrm{S}$, then $\mathcal{S}_{\mathrm{X}}^{\mathrm{c}}$ is a soft $C$-interior ideal of $\mathrm{S}$.

Proof. Let $X$ be $C$-interior ideal and $x=m n p \in X$. Since $X \subseteq S(S-X) S$, then $x=m n p \in S(S-X) S$, implying that $m, p \in S$ and $n \in S-X$. In this statement, $\mathcal{S}_{X}^{c}(m n p) \subseteq\left(\mathbb{S}-\mathcal{S}_{X}^{c}\right)(n)$. In fact,

$$
\begin{aligned}
\emptyset & =\mathcal{S}_{X}^{c}(m n p) \\
& \subseteq\left(\mathbb{S}-\mathcal{S}_{X}^{c}\right)(n) \\
& =\mathrm{U}-\mathrm{U} \\
& =\emptyset .
\end{aligned}
$$

Theorem 22 Let $\mathrm{f}_{\mathrm{S}}$ be a soft set over $\mathrm{U}$. Then, $\mathrm{f}_{\mathrm{S}}$ is a soft AC-interior ideal over $\mathrm{U}$ of $\mathrm{S}$ if and only if $\mathrm{f}_{\mathrm{S}}^{\mathrm{c}}$ is a soft C-interior ideal over $\mathrm{U}$ of $\mathrm{S}$.

Proof. Let $f_{S}$ is a soft AC-interior ideal over $U$ of $S$. In this statement,

$$
\begin{aligned}
f_{S}^{c}(x y z) & =\left(U-f_{S}\right)(x y z) \\
& \subseteq U-\left(\mathbb{S}-f_{S}\right)(y) \\
& =U-\left(U-f_{S}(y)\right) \\
& =U-f_{S}^{c}(y) \\
& =\left(\mathbb{S}-f_{S}^{c}\right)(y)
\end{aligned}
$$


for all $x, y, z \in S$. Conversely, let $f_{S}^{c}$ be a soft C-interior ideal over $U$ of $S$. Then,

$$
\begin{aligned}
f_{S}(x y z) & =\left(U-f_{S}^{c}\right)(x y z) \\
\supseteq & U-\left(\left(\mathbb{S}-f_{S}^{c}\right)(y)\right) \\
& =U-\left(U-f_{S}^{c}(y)\right) \\
& =U-f_{S}(y) \\
& =\left(\mathbb{S}-f_{S}\right)(y)
\end{aligned}
$$

for all $x, y, z \in S$. This completes the proof.

Theorem 23 Let $\mathrm{f}_{\mathrm{S}}$ be soft set over $\mathrm{U}$. Then, $\mathrm{f}_{\mathrm{S}}$ is a soft C-interior ideal over $\mathrm{U}$ if and only if

$$
\mathrm{f}_{\mathrm{S}} \widetilde{\simeq} \theta *\left(\mathbb{S}-\mathrm{f}_{\mathrm{S}}\right) * \theta
$$

Proof. Let $f_{S}$ be a soft C-interior ideal over $U$ and $x \in S$. If $f_{S}=\emptyset$, it is clear that $\mathrm{f}_{\mathrm{S}}(\mathrm{x}) \widetilde{\subseteq}\left(\theta *\left(\mathbb{S}-\mathrm{f}_{\mathrm{S}}\right) * \theta\right)(\mathrm{x})$, thus $\mathrm{f}_{\mathrm{S}} \widetilde{\widetilde{\subseteq}} \theta *\left(\mathbb{S}-\mathrm{f}_{\mathrm{S}}\right) * \theta$. If there exist elements $y, z, u, v$ of $S$ such that $x=y z$ and $y=m p$, we can write:

$$
f_{S}(x)=f_{S}(y z)=f_{S}(m p z) \subseteq\left(\mathbb{S}-f_{S}\right)(p) .
$$

Then,

$$
\begin{aligned}
\left(\theta *\left(\mathbb{S}-\mathrm{f}_{\mathrm{S}}\right) * \theta\right)(x) & =\left(\left(\theta *\left(\mathbb{S}-\mathrm{f}_{\mathrm{S}}\right)\right) * \theta\right)(\mathrm{x}) \\
& =\bigcap_{x=y z}\left(\theta *\left(\mathbb{S}-\mathrm{f}_{\mathrm{S}}\right)\right)(\mathrm{y}) \cup \theta(z) \\
& =\bigcap_{x=y z}\left[\bigcap_{y=m p}\left(\theta(\mathrm{m}) \cup\left(\mathbb{S}-\mathrm{f}_{\mathrm{S}}\right)(\mathrm{p})\right)\right] \cup \theta(z) \\
& =\bigcap_{x=y z} \bigcap_{y=m p}\left(\emptyset \cup\left(\mathbb{S}-\mathrm{f}_{\mathrm{S}}\right)(\mathrm{p})\right) \cup \emptyset \\
& \supseteq \bigcap_{x=y z} \bigcap_{y=m p} \emptyset \cup \mathrm{f}_{\mathrm{S}}(\mathrm{mpz}) \cup \emptyset \\
& =\mathrm{f}_{\mathrm{S}}(\mathrm{x}) .
\end{aligned}
$$

Thus, $\mathrm{f}_{\mathrm{S}} \widetilde{\simeq} \theta *\left(\mathbb{S}-\mathrm{f}_{\mathrm{S}}\right) * \theta$.

Conversely, accept that $\mathrm{f}_{\mathrm{S}} \widetilde{\subseteq} \theta *\left(\mathbb{S}-\mathrm{f}_{\mathrm{S}}\right) * \theta$ for $x, a, y, m, n, p, q \in S$. Then,

$$
\begin{aligned}
f_{S}(x a y) & \subseteq\left(\theta *\left(\mathbb{S}-f_{S}\right) * \theta\right)(x a y) \\
& =\bigcap_{x a y=m n}\left\{\left(\theta *\left(\mathbb{S}-f_{S}\right)\right)(m) * \theta(n)\right\} \\
& \subseteq\left(\theta *\left(\mathbb{S}-f_{S}\right)\right)(x a) \cup \theta(y) \\
& =\left(\theta *\left(\mathbb{S}-f_{S}\right)\right)(x a) \cup \emptyset \\
& =\bigcap_{x a=p q} \theta(p) \cup\left(\mathbb{S}-f_{S}\right)(q) \\
& \subseteq \theta(x) \cup\left(\mathbb{S}-f_{S}\right)(a) \\
& =\left(\mathbb{S}-f_{S}\right)(a)
\end{aligned}
$$

hence, $f_{\mathrm{S}}$ is a soft $\mathrm{C}$-interior ideal. This completes the proof. 
Theorem 24 Every soft union right $C$-Left ideal of $\mathrm{S}$ is a soft $C$-interior ideal of $\mathrm{S}$.

Proof. We accept that $f_{S}$ is a soft union right C-Left ideal. Then,

$$
\begin{aligned}
\left(\theta *\left(\mathbb{S}-\mathrm{f}_{\mathrm{S}}\right) * \theta\right)(\mathrm{mnp}) & =\left[\left(\theta *\left(\mathbb{S}-\mathrm{f}_{\mathrm{S}}\right)\right] * \theta\right)(\mathrm{mnp}) \\
& =\bigcap_{\mathrm{mnp}=\mathrm{uv}}\left[\left(\theta *\left(\mathbb{S}-\mathrm{f}_{\mathrm{S}}\right)\right](\mathrm{u}) \cup \theta(v)\right. \\
& =\bigcap_{\mathrm{mnp}=\mathrm{uv}}\left(\theta *\left(\mathbb{S}-\mathrm{f}_{\mathrm{S}}\right)\right)(\mathrm{u}) \\
& \supseteq \bigcap_{\mathrm{mnp}=\mathrm{uv}} \mathrm{f}_{\mathrm{S}}(\mathrm{u}) \\
& \supseteq \bigcap_{\mathrm{mnp}=\mathrm{uv}} \mathrm{f}_{\mathrm{S}}(\mathrm{uv}) \\
& =\mathrm{f}_{\mathrm{S}}(\mathrm{mnp})
\end{aligned}
$$

$f_{S}$ is a C-interior ideal of $S$.

Theorem 25 Let $\mathrm{f}_{\mathrm{S}}$ and $\mathrm{g}_{\mathrm{S}}$ be two soft C-interior ideals of $\mathrm{S}$ over $\mathrm{U}$. Then, $\mathrm{f}_{\mathrm{S}} \widetilde{\cap} \mathrm{g}_{\mathrm{S}}$ is a soft C-interior ideal over $\mathrm{U}$.

Proof. Let $m, n, p \in S$. Then,

$$
\begin{aligned}
\left(f_{S} \widetilde{\cap} g_{S}\right)(m n p) & =f_{S}(m n p) \cap g_{S}(m n p) \\
& \subseteq\left(\mathbb{S}-f_{S}\right)(n) \cap\left(\mathbb{S}-g_{S}\right)(n) \\
& \subseteq\left(\mathbb{S}-f_{S}\right)(n) \cup\left(\mathbb{S}-g_{S}\right)(n) \\
& =\left(\mathbb{S}-\left(f_{S} \widetilde{\cap} g_{S}\right)\right)(n)
\end{aligned}
$$

thus, $f_{S} \widetilde{\cap} g_{S}$ is a soft C-interior ideal over $U$. Now, we show that if $f_{S}$ and $g_{S}$ are two soft C-interior ideals of $S$ over $U, f_{S} \widetilde{U} g_{S}$ is not a soft C-interior ideal with the following example.

Example 9 Let $\mathbb{Z}_{4}$ be the semigroup over $\mathrm{U}=\mathrm{D}_{2}=\{e, x, y, y x\}$ in Example 6. Let the soft set $\mathrm{f}_{\mathrm{S}}$ and $\mathrm{g}_{\mathrm{S}}$ over $\mathrm{U}$ be as following: $\mathrm{f}_{\mathrm{S}}(0)=\emptyset, \mathrm{f}_{\mathrm{S}}(1)=\{e, x\}$, $f_{S}(2)=\{x\}, f_{S}(3)=\{y, y x\}$ and $g_{S}(0)=\emptyset, g_{S}(1)=\{e\}, g_{S}(2)=\{y\}, g_{S}(3)=$ $\{x, y, y x\}$. We see that $\left(f_{S} \widetilde{\cup} g_{S}\right)(333)=\left(f_{S} \widetilde{\cup} g_{S}\right)(1) \nsubseteq \mathbb{S}-\left(f_{S} \widetilde{\cup} g_{S}\right)(3)$.

Definition 17 A soft set $\mathrm{f}_{\mathrm{S}}$ over $\mathrm{U}$ is defined soft semi prime, if for all $a \in S$,

$$
f_{S}(a) \subseteq f_{S}\left(a^{2}\right)
$$

Proposition 12 Let $\mathrm{f}_{\mathrm{S}}$ be soft semi prime $C$-interior ideal of a semigroup $\mathrm{S}$. Then, $\mathrm{f}_{\mathrm{S}}\left(\mathrm{a}^{\mathfrak{n}}\right) \subseteq\left(\mathbb{S}-\mathrm{f}_{\mathrm{S}}\right)\left(\mathrm{a}^{\mathfrak{n}+1}\right)$ for all positive integers $\mathrm{n}$. 
Proof. Let $n$ be any positive integer. Then,

$$
f_{S}\left(a^{n}\right) \subseteq f_{S}\left(a^{2 n}\right) \subseteq f_{S}\left(a^{4 n}\right)=f_{S}\left(a^{3 n-2} a^{n+1} a\right) \subseteq\left(\mathbb{S}-f_{S}\right)\left(a^{n+1}\right) .
$$

Proposition 13 Let $\mathrm{f}_{\mathrm{S}}$ and $\mathrm{h}_{\mathrm{S}}$ be soft sets over $\mathrm{U}$ and $\Psi$ is a semigroup isomorphism from $\mathrm{S}$ to $\mathrm{T}$. If $\mathrm{f}_{\mathrm{S}}$ is a soft $C$-interior ideal of $\mathrm{S}$ over $\mathrm{U}$, then so is $\Psi\left(\mathrm{f}_{\mathrm{S}}\right)$ of $\mathrm{T}$ over $\mathrm{U}$.

Proposition 14 Let $\mathrm{f}_{\mathrm{S}}$ and $\mathrm{h}_{\mathrm{S}}$ be soft sets over $\mathrm{U}$ and $\Psi$ is a semigroup homomorphism from $\mathrm{S}$ to $\mathrm{T}$. If $\mathrm{f}_{\mathrm{S}}$ is a soft $C$-interior ideal of $\mathrm{T}$ over $\mathrm{U}$, then so is $\Psi^{-1}\left(\mathrm{f}_{\mathrm{T}}\right)$ of $\mathrm{S}$ over $\mathrm{U}$.

\subsection{Soft C- quasi ideals of semigroups}

In this subsection, we introduce soft covered quasi-ideals of semigroups, define their basic properties with respect to soft set operations, soft intersection product and certain kinds of soft C-ideals.

Definition 18 A soft set $\mathrm{f}_{\mathrm{S}}$ over $\mathrm{U}$ is called a soft covered quasi-ideal of $\mathrm{S}$ over $\mathrm{U}$ if

$$
\mathrm{f}_{\mathrm{S}} \widetilde{\widetilde{\subseteq}}\left(\left(\mathbb{S}-\mathrm{f}_{\mathrm{S}}\right) * \theta\right) \widetilde{\cup}\left(\theta *\left(\mathbb{S}-\mathrm{f}_{\mathrm{S}}\right)\right) .
$$

For the sake of brevity, soft covered quasi-ideal of $\mathrm{S}$ is denoted by soft C-quasiideal.

Proposition 15 Every soft $C$-quasi-ideal of $\mathrm{S}$ is a soft $C$-semigroup of $\mathrm{S}$.

Proof. We accept that $f_{S}$ is a soft C-quasi-ideal of $S$. Then, since $\theta \subseteq\left(\mathbb{S}-f_{S}\right)$,

$$
\theta *\left(\mathbb{S}-f_{S}\right) \widetilde{\subseteq}\left(\mathbb{S}-f_{S}\right) *\left(\mathbb{S}-f_{S}\right)
$$

and

$$
\left(\mathbb{S}-\mathrm{f}_{\mathrm{S}}\right) * \theta \widetilde{\widetilde{C}}\left(\mathbb{S}-\mathrm{f}_{\mathrm{S}}\right) *\left(\mathbb{S}-\mathrm{f}_{\mathrm{S}}\right)
$$

from here

$$
\mathrm{f}_{\mathrm{S}} \widetilde{\subseteq}\left(\theta *\left(\mathbb{S}-\mathrm{f}_{\mathrm{S}}\right)\right) \widetilde{\cup}\left(\left(\mathbb{S}-\mathrm{f}_{\mathrm{S}}\right) * \theta\right) \widetilde{\subseteq}\left(\mathbb{S}-\mathrm{f}_{\mathrm{S}}\right) *\left(\mathbb{S}-\mathrm{f}_{\mathrm{S}}\right)
$$

since $f_{S}$ is a soft $C$-quasi-ideal of $S$. Hence, $f_{S}$ is a soft C-semigroup of $S$.

Theorem 26 If $\mathrm{X}$ is a C-quasi-ideal of $\mathrm{S}$, then $\mathcal{S}_{\mathrm{X}}^{\mathrm{c}}$ is a soft $C$-quasi-ideal of $\mathrm{S}$. 
Proof. Let $X$ be a C-quasi-ideal and $x=m n \in X$. Since $X \subseteq((S-X) S) \cup$ $(S(S-X))$, then it is clear that $x=m n \in((S-X) S) \cup(S(S-X))$, implying that $m n \in(S-X) S$ or $m n \in S(S-X)$ and so $m \in(S-X)$ and $n \in S$ or $m \in S$ and $n \in(S-X)$. Hence since $\mathcal{S}_{X}^{\mathrm{c}}(\mathrm{X})=\mathcal{S}_{\mathbf{X}}^{\mathrm{c}}(\mathrm{mn})=\emptyset$, in any case

$$
\mathcal{S}_{X}^{\mathrm{c}} \widetilde{\widetilde{\subseteq}}\left(\left(\mathbb{S}-\mathcal{S}_{X}^{\mathrm{c}}\right) * \theta\right) \widetilde{\cup}\left(\theta *\left(\mathbb{S}-\mathcal{S}_{X}^{\mathrm{c}}\right)\right) .
$$

In fact, if we consider $\left[\left(\mathbb{S}-\mathcal{S}_{X}^{\mathrm{c}}\right) * \theta\right) \widetilde{\cup}\left(\theta *\left(\mathbb{S}-\mathcal{S}_{X}^{\mathrm{c}}\right)\right](\mathrm{x})$ and if $\mathrm{m} \in(\mathrm{S}-\mathrm{X})$, then

$$
\begin{aligned}
\left(\left(\mathbb{S}-\mathcal{S}_{X}^{c}\right) * \theta\right)(x) & =\bigcap_{x=m n}\left(\mathbb{S}-\mathcal{S}_{X}^{c}\right)(m) \cup \theta(\mathrm{n}) \\
& =\emptyset
\end{aligned}
$$

and

$$
\begin{aligned}
\left(\theta *\left(\mathbb{S}-\mathcal{S}_{X}^{\mathrm{c}}\right)\right)(x) & =\bigcap_{\chi=m n} \theta(\mathrm{m}) \cup\left(\mathbb{S}-\mathcal{S}_{X}^{\mathrm{c}}\right)(\mathrm{n}) \\
& =\emptyset
\end{aligned}
$$

and so $\left[\left(\mathbb{S}-\mathcal{S}_{X}^{\mathcal{c}}\right) * \theta\right) \widetilde{\cup}\left(\theta *\left(\mathbb{S}-\mathcal{S}_{X}^{\mathcal{c}}\right)\right](x)=\emptyset \cup \emptyset=\emptyset$. Hence,

$$
\mathcal{S}_{X}^{\mathrm{c}} \widetilde{\widetilde{\subseteq}}\left(\left(\mathbb{S}-\mathcal{S}_{X}^{\mathrm{c}}\right) * \theta\right) \widetilde{\cup}\left(\theta *\left(\mathbb{S}-\mathcal{S}_{X}^{\mathrm{c}}\right)\right) .
$$

Also if $m \in X$, then

$$
\begin{aligned}
\left(\left(\mathbb{S}-\mathcal{S}_{X}^{\mathrm{c}}\right) * \theta\right)(\mathrm{x}) & =\bigcap_{\mathrm{x}=\mathrm{mn}}\left(\mathbb{S}-\mathcal{S}_{X}^{\mathrm{c}}\right)(\mathrm{m}) \cup \theta(\mathrm{n}) \\
& =\mathrm{u}
\end{aligned}
$$

and

$$
\begin{aligned}
\left(\theta *\left(\mathbb{S}-\mathcal{S}_{X}^{\mathrm{c}}\right)\right)(x) & =\bigcap_{x=\mathrm{mn}} \theta(\mathrm{m}) \cup\left(\mathbb{S}-\mathcal{S}_{X}^{\mathrm{c}}\right)(\mathrm{n}) \\
& =\mathrm{U}
\end{aligned}
$$

and so $\left[\left(\mathbb{S}-\mathcal{S}_{X}^{\mathfrak{c}}\right) * \theta\right) \widetilde{\cup}\left(\theta *\left(\mathbb{S}-\mathcal{S}_{X}^{\mathfrak{c}}\right)\right](x)=\mathrm{U} \cup \mathrm{U}=\mathrm{U}$. Hence,

$$
\mathcal{S}_{X}^{\mathrm{c}} \widetilde{\widetilde{\subseteq}}\left(\left(\mathbb{S}-\mathcal{S}_{X}^{\mathrm{c}}\right) * \theta\right) \widetilde{\cup}\left(\theta *\left(\mathbb{S}-\mathcal{S}_{X}^{\mathrm{c}}\right)\right) .
$$

Proposition 16 Every soft C-left (right) ideal of $\mathrm{S}$ is a soft $C$-quasi-ideal of $\mathrm{S}$.

Proof. We accept that $f_{S}$ is a soft C-Left ideal of $S$ over $U$ which is defined $f_{S} \subseteq\left(\theta *\left(\mathbb{S}-f_{S}\right)\right)$. In this statement, we have:

$$
\mathrm{f}_{\mathrm{S}} \widetilde{\widetilde{\subseteq}}\left(\theta *\left(\mathbb{S}-\mathrm{f}_{\mathrm{S}}\right)\right) \widetilde{\subseteq}\left(\left(\mathbb{S}-\mathrm{f}_{\mathrm{S}}\right) * \theta\right) \widetilde{\cup}\left(\theta *\left(\mathbb{S}-\mathrm{f}_{\mathrm{S}}\right)\right)
$$

hence, $f_{S}$ is a soft $\mathrm{C}$-quasi-ideal.

The converse of the above proposition does not hold in general as shown in the table. 
Example 10 Think $S=\mathbb{Z}_{4}=\{0,1,2,3\}$ defined by the following table:

\begin{tabular}{l|llll}
$\cdot$ & 0 & 1 & 2 & 3 \\
\hline 0 & 0 & 0 & 0 & 0 \\
1 & 0 & 0 & 0 & 0 \\
2 & 0 & 0 & 0 & 0 \\
3 & 0 & 0 & 1 & 1
\end{tabular}

Let $\mathrm{f}_{\mathrm{S}}$ be a soft set over $\mathrm{U}=\mathrm{D}_{2}=\{\boldsymbol{e}, \mathrm{x}, \mathrm{y}, \mathrm{yx}\}$ and is defined as following, $f_{S}(0)=\emptyset, f_{S}(1)=\{e\}, f_{S}(2)=\{e\}, f_{S}(3)=\{y, y x\}$ and so $\left(\mathbb{S}-f_{S}\right)(0)=$ $\mathrm{U},\left(\mathbb{S}-f_{S}\right)(1)=\{x, y, y x\},\left(\mathbb{S}-f_{S}\right)(2)=\{x, y, y x\},\left(\mathbb{S}-f_{S}\right)(3)=\{e, x\}$. Then, one can show that $\left.\mathrm{f}_{\mathrm{S}}(\mathbf{1}) \subseteq\left(\left(\mathbb{S}-\mathrm{f}_{\mathrm{S}}\right) * \theta\right)\right)(1) \cup\left(\theta *\left(\mathbb{S}-\mathrm{f}_{\mathrm{S}}\right)\right)(1)=$ $\left(\mathbb{S}-f_{S}\right)(3) \cup\left(\left(\mathbb{S}-f_{S}\right)(3) \cap\left(\mathbb{S}-f_{S}\right)(2)\right)=\left(\mathbb{S}-f_{S}\right)(3)=\{e, x\}$ is a soft $C$ quasi-ideal but since $\mathrm{f}_{\mathrm{S}}(3.2)=\mathrm{f}_{\mathrm{S}}(1) \nsubseteq\left(\mathbb{S}-\mathrm{f}_{S}\right)(2), \mathrm{f}_{\mathrm{S}}$ is not a soft C-Left ideal.

Proposition 17 Every soft $C$-quasi-ideal is a soft $C$-bi-ideal of S.

Proof. Let $f_{S}$ be a soft C-quasi-ideal of $S$. Then,

$$
\begin{aligned}
f_{S} & \subseteq\left(\left(\mathbb{S}-f_{S}\right) * \theta\right) \widetilde{\cup}\left(\theta *\left(\mathbb{S}-f_{S}\right)\right) \\
& \subseteq\left(\left(\mathbb{S}-f_{S}\right) *(\theta * \theta)\right) \widetilde{\cup}\left((\theta * \theta) *\left(\mathbb{S}-f_{S}\right)\right) \\
& \left.\subseteq\left(\left(\mathbb{S}-f_{S}\right) * \theta *\left(\mathbb{S}-f_{S}\right)\right) \widetilde{U}\left(\left(\mathbb{S}-f_{S}\right) * \theta\right) *\left(\mathbb{S}-f_{S}\right)\right) \\
& =\left(\left(\mathbb{S}-f_{S}\right) * \theta *\left(\mathbb{S}-f_{S}\right)\right) \widetilde{\cup}\left(\left(\mathbb{S}-f_{S}\right) * \theta *\left(\mathbb{S}-f_{S}\right)\right) \\
& =\left(\mathbb{S}-f_{S}\right) * \theta *\left(\mathbb{S}-f_{S}\right)
\end{aligned}
$$

Hence, $\mathrm{f}_{\mathrm{S}}$ is a soft C-bi-ideal of $\mathrm{S}$ together with Proposition 15. The converse of this proposition does not hold in general as shown in the following example.

Example 11 Let $\mathrm{S}=\{\mathrm{a}, \mathrm{b}, \mathrm{c}, \mathrm{d}\}$ be the semigroup in Example 6 and $\mathrm{U}=$ $\mathrm{D}_{2}=\{e, x, y, y x\}$. Let $\mathrm{f}_{\mathrm{S}}$ be a soft set over $\mathrm{U}$ defined by $\mathrm{f}_{\mathrm{S}}(0)=\emptyset, \mathrm{f}_{\mathrm{S}}(1)=$ $\{x\}, f_{S}(2)=\{x, y\}, f_{S}(3)=\{y x\}$ and so $\left(\mathbb{S}-f_{S}\right)(0)=\mathrm{U},\left(\mathbb{S}-f_{S}\right)(1)=$ $\{e, y, y x\},\left(\mathbb{S}-f_{S}\right)(2)=\{e, y x\},\left(\mathbb{S}-f_{S}\right)(3)=\{e, x, y\}$. Then, $f_{S}$ is a soft C-biideals. However, $\mathrm{f}_{\mathrm{S}}$ is not a soft C-quasi-ideal since $\mathrm{f}_{\mathrm{S}}(1)=\{\mathrm{x}\} \nsubseteq\left(\left(\mathbb{S}-\mathrm{f}_{\mathrm{S}}\right) *\right.$ $\theta))(1) \cup\left(\theta *\left(\mathbb{S}-f_{S}\right)\right)(1)=\left(\mathbb{S}-f_{S}\right)(2) \cap\left(\mathbb{S}-f_{S}\right)(3)=\{e\}$.

Proposition 18 Let $\mathrm{f}_{\mathrm{S}}$ and $\mathrm{g}_{\mathrm{S}}$ be any soft $C$-Right ideal and soft $C$-Left ideal of $\mathrm{S}$ over $\mathrm{U}$, respectively. Then, $\mathrm{f}_{\mathrm{S}} \widetilde{\cap} \mathrm{g}_{\mathrm{S}}$ is a soft $C$-quasi-ideal. 
Proof. Let $f_{S}$ be any soft C-Right ideal of $S$ and $g_{S}$ be any soft C-Left ideal of $S$. Then,

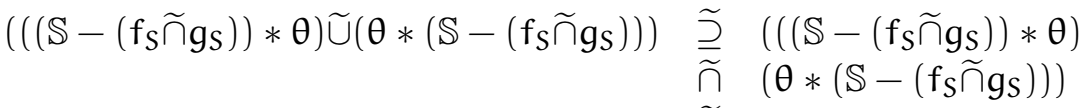

$$
\begin{aligned}
& \widetilde{\widetilde{\Omega}}\left(\left(\mathbb{S}-f_{S}\right) * \theta\right) \widetilde{\cap}\left(\theta *\left(\mathbb{S}-g_{S}\right)\right)
\end{aligned}
$$

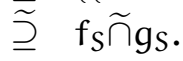

Thus, $f_{S} \widetilde{\cap} g_{S}$ is a soft C-quasi-ideal of $S$ over $U$.

Proposition 19 Let $\mathrm{f}_{\mathrm{S}}$ and $\mathrm{g}_{\mathrm{S}}$ be any soft $C$-quasi-ideals of $\mathrm{S}$ over $\mathrm{U}$. Then, $\mathrm{f}_{\mathrm{S}} \widetilde{\cap} \mathrm{g}_{\mathrm{S}}$ is a soft $C$-quasi-ideal .

Proof. Let $f_{S}$ and $g_{S}$ be any soft C-quasi-ideals of $S$. Then,

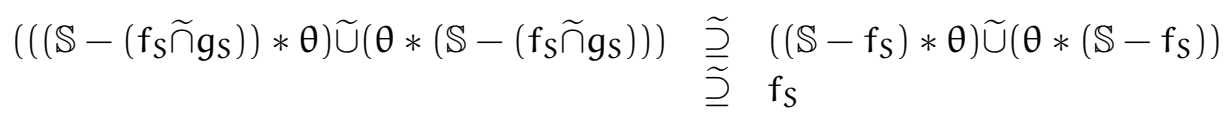

and

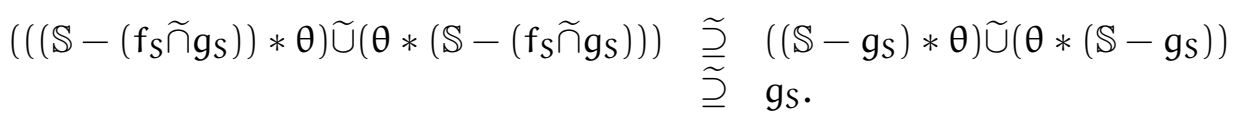

Thus, $\left(\left(\left(\mathbb{S}-\left(f_{S} \widetilde{\cap} g_{S}\right)\right) * \theta\right) \widetilde{\cup}\left(\theta *\left(\mathbb{S}-\left(f_{S} \widetilde{\cap} g_{S}\right)\right)\right) \widetilde{\supseteq}\left(f_{S} \widetilde{\cap} g_{S}\right)\right.$. Then, $f_{S} \widetilde{\cap} g_{S}$ is a soft C-quasi-ideal.

Proposition 20 Let $\mathrm{f}_{\mathrm{S}}$ be any soft $C$-quasi-ideal of a commutative semigroup $S$ and $\mathrm{a} \in \mathrm{A}$. Then,

$$
f_{S}\left(a^{n+1}\right) \subseteq\left(\mathbb{S}-f_{S}\right)\left(a^{n}\right)
$$

for every positive integer $\mathrm{n}$.

Proof. For any positive integer $n$, we have:

$$
\begin{aligned}
\left(\left(\mathbb{S}-f_{S}\right) * \theta\right)\left(a^{n+1}\right) & =\bigcap_{a^{n+1}=x y}\left(\mathbb{S}-f_{S}\right)(x) \cup \theta(y) \\
& \subseteq\left(\mathbb{S}-f_{S}\right)\left(a^{n}\right) \cup \emptyset \\
& =\left(\mathbb{S}-f_{S}\right)\left(a^{n}\right)
\end{aligned}
$$

from here $\left(\left(\mathbb{S}-f_{S}\right) * \theta\right)\left(a^{n+1}\right) \subseteq\left(\mathbb{S}-f_{S}\right)\left(a^{n}\right)$.

Moreover, since $f_{S}$ is a soft C-quasi-ideal of $S$, we have:

$$
\begin{aligned}
f_{S}\left(a^{n+1}\right) & \subseteq\left[\left(\left(\mathbb{S}-f_{S}\right) * \theta\right) \widetilde{\cup}\left(\theta *\left(\mathbb{S}-f_{S}\right)\right)\right]\left(a^{n+1}\right) \\
& =\left(\left(\mathbb{S}-f_{S}\right) * \theta\right)\left(a^{n+1}\right) \cup\left(\theta *\left(\mathbb{S}-f_{S}\right)\right)\left(a^{n+1}\right) \\
& \subseteq\left(\mathbb{S}-f_{S}\right)\left(a^{n}\right) \cup\left(\mathbb{S}-f_{S}\right)\left(a^{n}\right) \\
& =\left(\mathbb{S}-f_{S}\right)\left(a^{n}\right) .
\end{aligned}
$$

This completes the proof. 


\subsection{Soft C-generalized Bi-ideals of semigroups}

In this subsection, we study soft covered generalized bi-ideals of semigroups, introduce their basic prosperities as regards soft set operations, soft intersection product and certain kinds of soft C-ideals.

Definition 19 A soft set over $\mathrm{U}$ is called a soft covered generalized bi-ideal of $S$, if

$$
f_{S}(x y z) \subseteq\left(\mathbb{S}-f_{S}\right)(x) \cup\left(\mathbb{S}-f_{S}\right)(z)
$$

for all $x, y, z \in S$.

For the sake of brevity, soft covered generalized bi-ideal is denoted by soft C-generalized bi-ideal of S. Clearly, every soft C-bi-ideal of $\mathrm{S}$ is a soft Cgeneralized bi-ideal of $\mathrm{S}$ but converse of this statement is not true. This is indicated by following example.

Example 12 Let think the semigroup $S=\{0,1,2,3\}$ in Example 6 and define the soft set $\mathrm{f}_{\mathrm{S}}$ over $\mathrm{U}=\mathrm{D}_{4}$ such that $\mathrm{f}_{\mathrm{S}}(0)=\emptyset, \mathrm{f}_{\mathrm{S}}(1)=\left\{x, x^{2}, \mathrm{y}\right\}, \mathrm{f}_{\mathrm{S}}(2)=$ $\{x, y x\}, f_{S}(3)=\left\{e, y x, y x^{2}\right\}$ and so $\left(\mathbb{S}-f_{S}\right)(0)=\left\{e, x, x^{2}, y, y x, y x^{2}\right\}$, ( $\mathbb{S}-$ $\left.f_{S}\right)(1)=\left\{e, y x, y x^{2}\right\},\left(\mathbb{S}-f_{S}\right)(2)=\left\{e, y, x^{2}, y x^{2}\right\},\left(\mathbb{S}-f_{S}\right)(3)=\left\{x, y, x^{2}\right\}$.

Then, one can easily show that $\mathrm{f}_{\mathrm{S}}$ is a soft $C$-generalized bi-ideal of $\mathrm{S}$ over U. However, since $\mathrm{f}_{\mathrm{S}}(33)=\mathrm{f}_{\mathrm{S}}(2) \nsubseteq\left(\mathbb{S}-\mathrm{f}_{\mathrm{S}}\right)(3) \cup\left(\mathbb{S}-\mathrm{f}_{\mathrm{S}}\right)(3)$, $\mathrm{f}_{\mathrm{S}}$ is not a soft $C$-bi-ideal of $\mathrm{S}$.

Theorem 27 If $\mathrm{X}$ is a $C$-generalized bi-ideal of $\mathrm{S}$, then $\mathcal{S}_{\mathrm{X}}^{\mathrm{c}}$ is a soft $C$-generalized bi-ideal of $\mathrm{S}$.

Theorem 28 Let $\mathrm{f}_{\mathrm{S}}$ be a soft set over $\mathrm{U}$. Then, $\mathrm{f}_{\mathrm{S}}$ is a soft C-generalized bi-ideal over $\mathrm{U}$ of $\mathrm{S}$ if and only if $\mathrm{f}_{\mathrm{S}}^{\mathcal{c}}$ is a soft $C$-generalized bi-ideal over $\mathrm{U}$ of $\mathrm{S}$.

Theorem 29 Let $\mathrm{f}_{\mathrm{S}}$ be a soft set over $\mathrm{U}$. Then, $\mathrm{f}_{\mathrm{S}}$ is a soft C-generalized bi-ideal of $\mathrm{S}$ over $\mathrm{U}$ if and only if

$$
\mathrm{f}_{\mathrm{S}} \widetilde{\subseteq}\left(\mathbb{S}-\mathrm{f}_{\mathrm{S}}\right) * \theta *\left(\mathbb{S}-\mathrm{f}_{\mathrm{S}}\right) .
$$

Theorem 30 Every soft C-left (right) ideal of a semigroup $\mathrm{S}$ over $\mathrm{U}$ is a soft $C$-generalized bi-ideal of $\mathrm{S}$ over $\mathrm{U}$.

Proof. Let $\mathrm{f}_{\mathrm{S}}$ be a soft C-left (right) ideal of $\mathrm{S}$ over $\mathrm{U}$ and $x, y, z \in \mathrm{S}$. Then,

$$
f_{S}(x y z)=f_{S}((x y) z) \subseteq\left(\mathbb{S}-f_{S}\right)(z) \subseteq\left(\mathbb{S}-f_{S}\right)(x) \cup\left(\mathbb{S}-f_{S}\right)(z)
$$

Thus, $f_{S}$ is a soft $\mathrm{C}$-generalized bi-ideal of $S$. 
Proposition 21 Let $\mathrm{f}_{\mathrm{S}}$ and $\mathrm{f}_{\mathrm{T}}$ be soft $C$-generalized bi-ideals of $\mathrm{S}$ over $\mathrm{U}$. Then, $\mathrm{f}_{\mathrm{S}} \widetilde{\mathrm{f}_{\mathrm{T}}}$ is a soft C-generalized bi-ideal of $\mathrm{S} \times \mathrm{T}$ over $\mathrm{U}$.

Proposition 22 Let $\mathrm{f}_{\mathrm{S}}$ and $\mathrm{h}_{\mathrm{S}}$ be soft sets over $\mathrm{U}$ and $\Psi$ is a semigroup isomorphism from $\mathrm{S}$ to $\mathrm{T}$. If $\mathrm{f}_{\mathrm{S}}$ is a soft $C$-generalized bi-ideal of $\mathrm{S}$ over $\mathrm{U}$, then so is $\Psi\left(\mathrm{f}_{\mathrm{S}}\right)$ of $\mathrm{T}$ over $\mathrm{U}$.

Proposition 23 Let $\mathrm{f}_{\mathrm{S}}$ and $\mathrm{h}_{\mathrm{S}}$ be soft sets over $\mathrm{U}$ and $\Psi$ is a semigroup homomorphism from $\mathrm{S}$ to $\mathrm{T}$. If $\mathrm{f}_{\mathrm{T}}$ is a soft $C$-generalized bi-ideal of $\mathrm{T}$ over $\mathrm{U}$, then so is $\Psi^{-1}\left(\mathrm{f}_{\mathrm{T}}\right)$ of $\mathrm{S}$ over $\mathrm{U}$.

\section{Conclusion}

In this manuscript, we have introduced soft C-semigroups, C-left (right) ideals, C-bi-ideals, C-interior ideals, C-quasi-ideals and C-generalized bi-ideals. Moreover, we survey the relation between soft AC-ideals and soft C-ideals. Addition to, we obtain the interrelations of various soft $\mathrm{C}$-ideals as in the following figure.

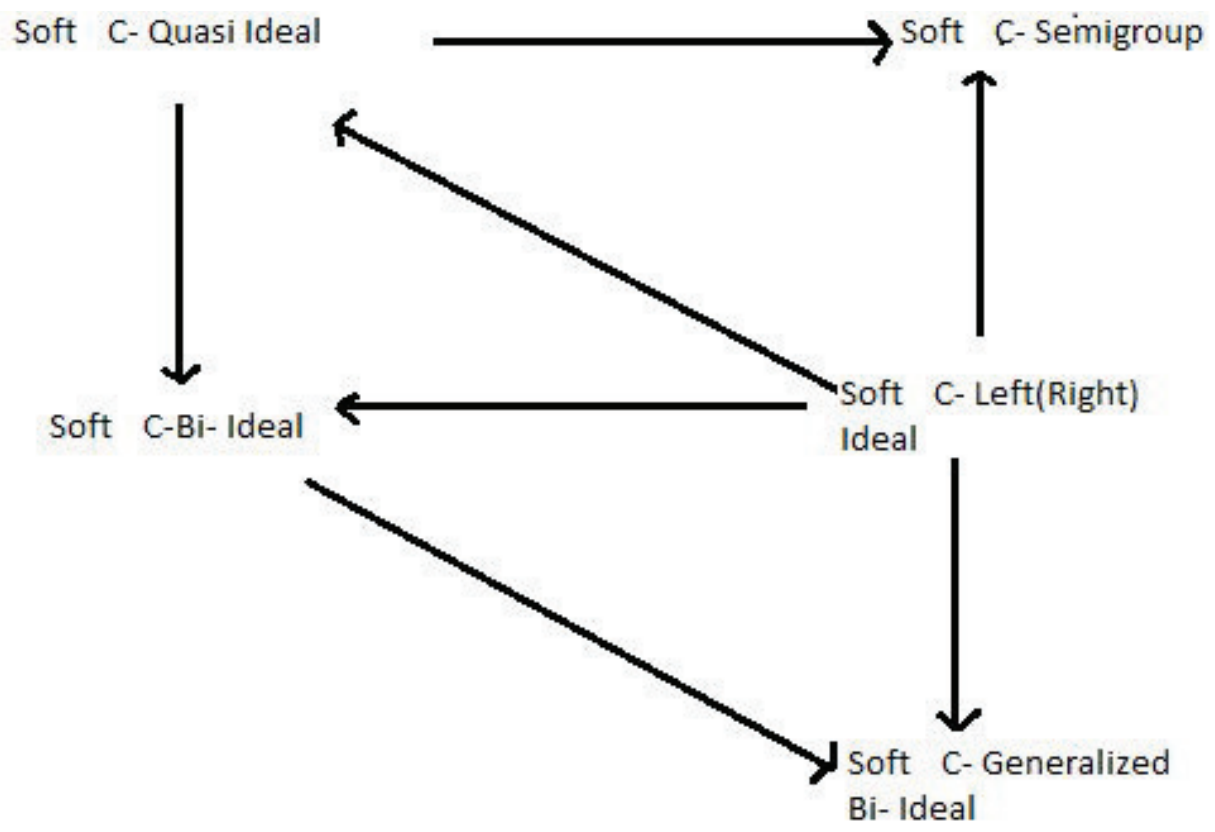

Figure 1 


\section{References}

[1] D. Molodtsov, Soft set theory-first results, Comput Math Appl., 37 (1999), $19-31$.

[2] H. Aktas and N. Cağman, Soft sets and soft groups, Inform Sci., 177 (2007), 2726-2735.

[3] F. Feng, Y. B. Jun and X. Zhao, Soft semirings, Comput Math Appl., 56 (2008), 2621-2628.

[4] U. Acar, F. Koyuncu and B. Tanay, Soft sets and soft rings, Comput Math Appl, 59 (2010), 3458-3463.

[5] Y. B. Jun, Soft BCK/BCI-algebras, Comput Math Appl., 56 (2008), 14081413.

[6] Y. B. Jun and C. H. Park, Applications of soft sets in ideal theory of BCK/BCI-algebras, Inform Sci., 178 (2008), 2466-2475.

[7] Y. B. Jun, K. J. Lee and J. Zhan, Soft p-ideals of soft BCI-algebras, Comput Math Appl., 58 2009, 2060-2068.

[8] Y. B. Jun, K. J. Lee, C. H. Park, Soft set theory applied to ideals in d-algebras, Comput. Math. Appl., 57 (3) (2009), 367-378.

[9] J. Zhan, Y. B. Jun, Soft BL-algebras based on fuzzy sets, Comput Math Appl., 59 (6) (2010), 2037-2046.

[10] O. Kazancı, Ş. Yılmaz and S. Yamak, Soft sets and soft BCH-algebras, Hacet J Math Stat., 39 (2) (2010), 205-217.

[11] A. Sezgin and A. O. Atagün and E. Aygün, A note on soft N-groups and idealistic soft N-groups, Filomat, 25 (1), 53-68.

[12] N. C̣ăgman and S. Enginoğlu, Soft matrix theory and its decision making, Comput Math Appl., 59 (2010), 3308-3314.

[13] N. C̣ağman and S. Enginoğlu, Soft set theory and uni-int decision making, Eur J Oper Res., 207 (2010), 848-855.

[14] P. K. Maji, A. R. Roy and R. Biswas, An application of soft sets in a decision making problem, Comput Math Appl., 44 (2002), 1077-1083. 
[15] Y. Zou and Z. Xiao, Data analysis approaches of soft sets under incomplete information, Knowled-Based Syst., 21 (2008), 941-945.

[16] D. Mandal, Fuzzy ideals and fuzzy interior idelas in ordered semigroups, In Fuzzy Information and Engineering, (2014), 101-114.

[17] K. A. Dib and N. Galhum, Fuzzy ideals and fuzzy bi-ideals in fuzzy semigroups, Fuzzy Sets and Systems, 92 (1992), 103-111.

[18] O. Kazancý and S. Yamak, Generalized fuzzy bi-ideals of semigroup, Soft Computing, 12 (2008), 1119-1124.

[19] J. Kavikumar, A. B. Khamis, Fuzzy Ideals and Fuzzy Quasi-ideals in Ternary Semirings, IAENG International Journal of Applied Mathematics, 37 (2) (2007).

[20] T. Changphas and P. Summaprab, On Ordered Semigroups Containing Covered Ideals, Communications in Algebra, 44 (9) (2016), 4104-4113, DOI: $10.1080 / 00927872.2015 .1087015$.

[21] A. S. Sezgin, N. C̣ağman, A. O. Atagün, M. I. Ali, Türkmen E. Soft intersection semigroups, ideals and bi-ideals; a new application on semigroup theory I, Filomat, 29 (5) (2015), 917-946.

[22] A. S. Sezgin, N. C̣ă̆man, A. O. Atagün, Soft intersection interior ideals, quasi-ideals and generalized bi-ideals; a new approach to semigroup theory II, Journal of Multiple-Valued Logic and Soft Computing, 23 (1-2) (2014), 161-207.

[23] I. Fabrici, Semigroups containing covered one-sided ideals, Mahematica Slovaca, 31 (1981), 225-231.

[24] I. Fabrici, Semigroups containing covered two-sided ideals, Mahematica Slovaca, 34 (1984), 355-363.

[25] X., Xie, F. Yan, Fuzzy Anti-Covered Left Ideals in Semigroups, submitted.

[26] A. Sezgin, Ş Özlü, Soft Anti-Covered Ideals in Semigroups, submitted.

[27] N. Çă̆man, F. Çıtak and H. Aktaş, Soft int-groups and its applications to group theory, Neural Comput. Appl., 21 (1) (2012), 151-158. 